TTK $-11-18$

\title{
A Bayesian view of the current status of dark matter direct searches
}

\author{
Chiara Arina†, Jan Hamann $\ddagger$ and Yvonne Y. Y. Wong† \\ † Institut für Theoretische Teilchenphysik und Kosmologie, RWTH Aachen, 52056 \\ Aachen, Germany \\ $\ddagger$ Department of Physics and Astronomy, University of Aarhus, 8000 Aarhus C, \\ Denmark \\ E-mail: chiara.arina@physik.rwth-aachen.de, hamann@phys.au.dk, \\ yvonne.wong@physik.rwth-aachen.de
}

\begin{abstract}
Bayesian statistical methods offer a simple and consistent framework for incorporating uncertainties into a multi-parameter inference problem. In this work we apply these methods to a selection of current direct dark matter searches. We consider the simplest scenario of spin-independent elastic WIMP scattering, and infer the WIMP mass and cross-section from the experimental data with the essential systematic uncertainties folded into the analysis. We find that when uncertainties in the scintillation efficiency of Xenon100 have been accounted for, the resulting exclusion limit is not sufficiently constraining to rule out the CoGeNT preferred parameter region, contrary to previous claims. In the same vein, we also investigate the impact of astrophysical uncertainties on the preferred WIMP parameters. We find that within the class of smooth and isotropic WIMP velocity distributions, it is difficult to reconcile the DAMA and the CoGeNT preferred regions by tweaking the astrophysics parameters alone. If we demand compatibility between these experiments, then the inference process naturally concludes that a high value for the sodium quenching factor for DAMA is preferred.
\end{abstract}




\section{Introduction}

Recent years have seen a fervent activity in the direct search of Weakly Interacting Massive Particles (WIMPs) in the Galactic dark matter (DM) halo. Besides the wellestablished results of DAMA/NaI and DAMA/Libra [1], which have observed altogether 13 successive cycles of annual modulation in the nuclear recoil rate consistent with the signature of Galactic WIMP scattering, the CoGeNT experiment also claims an excess of events that cannot be accounted for by known background sources [2]. If interpreted as DM signals, then these results point to a particle mass of few $\mathrm{GeVs}$ in model independent analyses (e.g., [3-7]), as well as in the frameworks of scalar DM (e.g., [8,9]), supersymmetric models (e.g., [10-14]), and hidden sectors (e.g., [15, 16]).

Concurrently, the null results of several other direct detection experiments have led to exclusion limits in the WIMP parameter space. For spin-independent scattering, CDMS [17,18], Xenon100 [19], Xenon10 [20], Edelweiss [21], the CRESST run on Tungsten [22], and Zeplin-III [23] have all set relevant bounds. Most notably, the limits set by Xenon100 on the WIMP mass and cross-section appear to be incompatible with the regions preferred by the DM interpretation of the DAMA and CoGeNT results. Prudently though, we note that, depending on the detection techniques, direct WIMP searches can be subject to large systematic effects. Indeed, in the case of Xenon10, different choices of the scintillation efficiency $\mathrm{L}_{\text {eff }}$ can either enhance or reduce the compatibility between its exclusion limits and the DAMA/CoGeNT preferred parameters $[8,24,25]$.

The first goal of this work, therefore, is to address the issue of how to account for systematic uncertainties in direct detection experiments. To this end, we employ the techniques of Bayesian inference. Bayesian methods provide a simple and consistent framework for dealing with nuisance parameters - in this instance, poorly known experimental parameters such as $\mathrm{L}_{\text {eff }}$-in an inference problem. Once a likelihood function has been defined for an experimental result, the nuisance parameters can be systematically integrated out of the problem in a procedure known as marginalisation, yielding a final posterior probability density function (pdf) for the WIMP parameters that incorporates all relevant sources of uncertainties. Because the process of marginalisation requires the evaluation of a multi-dimensional integral, Markov Chain Monte Carlo (MCMC) methods are particularly well-suited to the purpose. Lastly, we note that Bayesian inference is widely used for parameter estimation in precision cosmology (e.g., [26]), and has recently also found application in high energy physics, e.g., for the exploration of supersymmetric parameter space (e.g., [27-29]), or in view of forecasting model expectations for direct DM searches [30-32].

In the same vein, the second goal of this work is to incorporate also into the picture some degree of uncertainty in the astrophysics. The WIMP-nucleus scattering rate in a direct DM search depends on the (unknown) velocity distribution of the DM particles in the Galactic halo. Because of its simplicity a common practice is to assume a Maxwellian distribution, in which the local DM density, the circular and 
the escape velocities are fixed at some "standard" values. However, these quantities are far from well-constrained by astrophysical observations. Of even less certainty is our knowledge of the functional form of the WIMP velocity distribution. Indeed, simulations of structure formation suggest that substantial deviations from the isotropic Maxwellian form are highly probable (e.g., [33-36]). In this work we investigate several alternative velocity distributions. For simplicity we consider only isotropic equilibrium distributions consistent with selected spherically symmetric, smooth parametric DM halo density profiles motivated by $N$-body simulations. Nevertheless, we see no obvious obstacle to generalising the analysis also to anisotropic velocity distributions (e.g., [37-42]) and non-smooth density profiles (e.g., [43-47]).

The rest of the paper is organised as follows: after reviewing the basics of direct DM searches in section 2, we describe in section 3 various halo profiles and their corresponding WIMP velocity distributions. In section 4 we construct the likelihood function for each experiment and discuss the modeling of their associated systematics. Section 5 contains a detailed explanation of the Bayesian inference procedure. We present our inference results in section 6 , and conclude in section 7 .

\section{The WIMP signal in direct detection experiments}

Direct detection experiments aim to detect or set limits on nuclear recoils arising from the scattering of WIMPs off target nuclei. The differential spectrum for such recoils, in units of events per time per detector mass per energy, has the form

$$
\frac{\mathrm{d} R}{\mathrm{~d} E}=\frac{\rho_{\odot}}{m_{\mathrm{DM}}} \int_{v^{\prime}>v_{\min }^{\prime}} \mathrm{d}^{3} v^{\prime} \frac{\mathrm{d} \sigma}{\mathrm{d} E} v^{\prime} f\left(\overrightarrow{v^{\prime}}(t)\right),
$$

where $E$ is the energy transferred during the collision, $\rho_{\odot} \equiv \rho_{\mathrm{DM}}\left(R_{\odot}\right)$ the WIMP density in the solar neighbourhood, $m_{\mathrm{DM}}$ the WIMP mass, $\mathrm{d} \sigma / \mathrm{d} E$ the differential cross section for the scattering, and $f\left(\overrightarrow{v^{\prime}}(t)\right)$ is the WIMP velocity distribution in the Earth's rest frame normalised such that $\int \mathrm{d}^{3} v^{\prime} f\left(\overrightarrow{v^{\prime}}(t)\right)=1$. The integration in the differential rate is performed over all incident particles capable of depositing a recoil energy of $E$. For

elastic scattering, this implies a lower integration limit of $v_{\text {min }}^{\prime}=\sqrt{M_{\mathcal{N}} E / 2 \mu}$, where $M_{\mathcal{N}}$ is the mass of the target nucleus, and $\mu=m_{\mathrm{DM}} M_{\mathcal{N}} /\left(m_{\mathrm{DM}}+M_{\mathcal{N}}\right)$ is the WIMP-nucleus reduced mass. We defer the discussion of the normalised velocity distribution $f\left(\overrightarrow{v^{\prime}}(t)\right)$ to section 3.

The differential cross-section $\mathrm{d} \sigma / \mathrm{d} E$ encodes all the particle and nuclear physics information. For coherent elastic scattering it is parameterised as

$$
\frac{\mathrm{d} \sigma}{\mathrm{d} E}=\frac{M_{\mathcal{N}} \sigma_{n}^{\mathrm{SI}}}{2 \mu_{n}^{2} v^{\prime 2}} \frac{\left(f_{p} Z+(A-Z) f_{n}\right)^{2}}{f_{n}^{2}} \mathcal{F}^{2}(E),
$$

where $\mu_{n}=m_{\mathrm{DM}} m_{n} /\left(m_{\mathrm{DM}}+m_{n}\right)$ is the WIMP-nucleon reduced mass, $\sigma_{n}^{\text {SI }}$ the spinindependent (SI) zero-momentum WIMP-nucleon cross-section, $Z(A)$ the atomic (mass) number of the target nucleus used, and $f_{p}\left(f_{n}\right)$ is the WIMP effective coherent coupling to the proton (neutron). We assume the WIMP couples equally to the neutron 
and the proton, so that the differential cross-section $\mathrm{d} \sigma / \mathrm{d} E$ is sensitive only to $A^{2}$. The nuclear form factor $\mathcal{F}(E)$ characterises the loss of coherence for nonzero momentum transfer, and in our analysis we use the Helm form factor [48, 49],

$$
F(E)=3 e^{-k^{2} s^{2} / 2} \frac{\sin (k r)-k r \cos (k r)}{(k r)^{3}},
$$

where $s=1 \mathrm{fm}, r=\sqrt{R^{2}-5 s^{2}}, R=1.2 A^{1 / 3} \mathrm{fm}$, and $k=\sqrt{2 M_{\mathcal{N}} E}$.

The total number of recoils expected in a detector of mass $M_{\text {det }}$ in a given observed energy range $\left[\mathcal{E}_{1}, \mathcal{E}_{2}\right]$ over an exposure time $T$ is obtained by integrating equation $(2.1)$ over energy,

$$
S(t)=M_{\mathrm{det}} T \int_{\mathcal{E}_{1} / q}^{\mathcal{E}_{2} / q} \mathrm{~d} E \epsilon(q E) \frac{\mathrm{d} R}{\mathrm{~d} E}
$$

where we have folded into the integral an energy-dependent function $\epsilon(q E)$ describing the efficiency of the detector. The quenching factor $q$, defined via $\mathcal{E}=q E$, denotes the fraction of recoil energy that is ultimately observed in a specific detection channel (scintillation or phonons/heat), and is a detector-dependent quantity. To distinguish $\mathcal{E}$ from the actual nuclear recoil energy $E$, the former is usually given in units of keVee (electron equivalent $\mathrm{keV}$ ), while the latter in $\mathrm{keVnr}$ (nuclear recoil keV).

\section{The WIMP velocity distribution}

\subsection{Halo profiles}

Two astrophysical factors enter into the differential recoil rate (2.1): the local WIMP density $\rho_{\odot}$ and the corresponding normalised velocity distribution $f\left(\overrightarrow{v^{\prime}}(t)\right)$ in the Earth's rest frame (primed $\overrightarrow{v^{\prime}}$ ). These quantities are related via

$$
\rho_{\mathrm{DM}}(\vec{r})=\int \mathrm{d}^{3} v F(\vec{v}, \vec{r}),
$$

where $\rho_{\mathrm{DM}}(\vec{r})$ is the WIMP density at $\vec{r}$ from the Galactic Centre (GC) such that $\rho_{\odot} \equiv \rho_{\mathrm{DM}}\left(\vec{R}_{\odot}\right)$ with $R_{\odot} \equiv\left|\vec{R}_{\odot}\right|=8.5 \mathrm{kpc}$, and $F(\vec{v}, \vec{r})$ is the WIMP velocity distribution in the Galactic frame (unprimed $\vec{v}$ ) whereby $f\left(\overrightarrow{v^{\prime}}(t)\right) \equiv F\left(\vec{v}, \vec{R}_{\odot}\right) / \rho_{\odot}$.

Most analyses in the literature assume a spherically symmetric and isothermal distribution for the WIMP around the GC. The WIMP velocities follow the Maxwellian distribution $F(\vec{v}, r) \sim \exp \left(-v^{2} / \bar{v}^{2}\right)$, where $\bar{v}=220 \mathrm{~km} \mathrm{~s}^{-1}$ is the mean velocity in the Galactic frame, and the distribution is cut off at $v>v_{\text {esc }}=544 \mathrm{~km} \mathrm{~s}^{-1}$. The resulting density profile scales as $r^{-2}$ [50], and is normalised to $\rho_{\odot}=0.3 \mathrm{GeV} \mathrm{cm}^{-3}$. This is known as the Standard Model Halo (SMH).

However, isothermal DM density profiles are rarely if ever encountered in $N$-body simulations. Indeed, most simulations find dark matter halos that are often "cuspy" and have density profiles that fall off faster than the $r^{-2}$ dependence of the SMH at large $r$. In view of the uncertainty in the exact DM distribution, we consider, besides the $\mathrm{SMH}$, four other spherically symmetric DM density profiles found in the literature: 
(i) Cored isothermal A variant of the SMH, this density profile has the form

$$
\rho_{\mathrm{DM}}(r)=\rho_{s}\left[1+\left(\frac{r}{r_{s}}\right)^{2}\right]^{-1} .
$$

Unlike the SMH in which the density $\rho_{\mathrm{DM}}(r)$ diverges as $r \rightarrow 0$, the cored isothermal halo has a finite density core whose size and density are characterised by the parameters $r_{s}$ and $\rho_{s}$ respectively. The profile's large $r$ (i.e., $r \gg r_{s}$ ) behaviour, however, is similarly to that of the SMH.

(ii) Navarro-Frenk-White (NFW) Based on N-body simulation results, Navarro, Frenk and White suggested as a universal form for the DM density profile across a wide range of halo masses $\left(10^{11} \rightarrow 10^{15} M_{\odot}\right)$ [51],

$$
\rho_{\mathrm{DM}}(r)=\rho_{s}\left(\frac{r}{r_{s}}\right)^{-1}\left(1+\left(\frac{r}{r_{s}}\right)\right)^{-2} .
$$

The density here falls off as $r^{-3}$ at $r \gg r_{s}$, while at $r \ll r_{s}$ we find a $r^{-1}$ behaviour (i.e., the cusp). The NFW profile is formally divergent as $r \rightarrow 0$. For numerical stability, however, we introduce in the profile a small core of size $\epsilon \ll r_{s}$. A related density profile is the Moore profile [52], which also exhibits a $r^{-3}$ behaviour at $r \gg r_{s}$, but has a steeper cusp that scales as $r^{-1.5}$. We do not consider the Moore profile here because of its similarity to the NFW profile (see section 3.2).

(iii) Einasto Some recent studies find that the Einasto profile [53] provides as good a fit as the NFW profile to DM halos found in $N$-body simulations of the concordance $\Lambda \mathrm{CDM}$ model [54]. The Einasto profile has the form

$$
\rho_{\mathrm{DM}}(r)=\rho_{s} \exp \left(-\frac{2}{a}\left[\left(\frac{r}{r_{s}}\right)^{a}-1\right]\right),
$$

where $a=0.17$, and its central density is finite.

(iv) Burkert The Burkert profile,

$$
\rho_{\mathrm{DM}}(r)=\rho_{s}\left(1+\frac{r}{r_{s}}\right)^{-1}\left(1+\frac{r}{r_{s}}\right)^{-2},
$$

is a cored profile that appears to provide a good fit to the DM distribution of dwarf galaxies [55].

All four profiles depend on two parameters $\rho_{s}$ and $r_{s}$. However, it is equally valid, and perhaps more enlightening, to adopt a parameterisation in terms of the virial mass $M_{\text {vir }}$ of the DM halo - defined as the mass contained in a sphere of radius $r_{\text {vir }}$ whose average density is 200 times the critical density - and a concentration parameter given by $c_{\text {vir }}=r_{\text {vir }} / r_{s}$. The advantage of this parameterisation is that, firstly, it is possible to specify directly a prior for $M_{\text {vir }}$ based on what we know about the mass of the Milky Way from satellite kinematics etc. Secondly, the concentration parameter $c_{\text {vir }}$ is well studied in $N$-body simulations, which again allow us to impose a prior on $c_{\text {vir }}$ in a meaningful way. We show how each density profile (3.2) to (3.5) can be expressed in terms of $M_{\text {vir }}$ and $c_{\text {vir }}$ in Appendix A. 


\subsection{Extracting the velocity distribution}

Given a DM density profile, the underlying DM velocity distribution can be extracted by inverting equation (3.1) under the assumption of hydrostatic equilibrium. For a spherically symmetric density distribution and assuming an isotropic velocity distribution $F(\vec{v}, r)=F(\varepsilon)$ in the Galactic frame that depends only on the relative energy $\varepsilon \equiv \Psi-\frac{1}{2} v^{2} \geq 0$ of the system, the solution is given by the Eddington formula [50],

$$
F(\varepsilon)=\frac{1}{\sqrt{8} \pi^{2}}\left[\int_{0}^{\varepsilon} \frac{\mathrm{d}^{2} \rho_{\mathrm{DM}}}{\mathrm{d} \Psi^{2}} \frac{\mathrm{d} \Psi}{\sqrt{\varepsilon-\Psi}}+\left.\frac{1}{\sqrt{\varepsilon}}\left(\frac{\mathrm{d} \rho_{\mathrm{DM}}}{\mathrm{d} \Psi}\right)\right|_{\Psi=0}\right] .
$$

The function $\Psi(r)$ is the gravitational potential generated by the DM halo and the baryonic matter residing in the Galactic disk and bulge, defined so that $\Psi(r \rightarrow \infty)=0$, and $v_{\text {esc }}(r) \equiv \sqrt{2 \Psi(r)}$ is the escape velocity at $r$. It is obtained by solving the Poisson equation,

$$
\frac{\mathrm{d}^{2} \Psi}{\mathrm{d} r^{2}}+\frac{2}{r} \frac{\mathrm{d} \Psi}{\mathrm{d} r}=-4 \pi G\left[\rho_{\mathrm{DM}}+\rho_{\text {disk }}+\rho_{\text {bulge }}\right],
$$

where the disk density distribution is given by [30]

$$
\rho_{\text {disk }}(r)=\frac{M_{\text {disk }}}{4 \pi r_{\text {disk }}^{2}} \frac{e^{-r / r_{\text {disk }}}}{r},
$$

with $M_{\text {disk }}=5 \times 10^{10} M_{\odot}$ and $r_{\text {disk }}=4 \mathrm{kpc}$, and the bulge is modelled as a point mass sitting at $\vec{r}=0$,

$$
\rho_{\text {bulge }}(r)=M_{\text {bulge }} \delta_{D}^{(3)}(\vec{r}),
$$

where $M_{\text {bulge }}=1.5 \times 10^{10} M_{\odot}$, and $\delta_{D}^{(3)}(\vec{r})$ is the 3-dimensional Dirac delta distribution.

At any given point $r$, the Eddington formula (3.6) returns a positive and nonzero solution for $F(\varepsilon)$ only up to the escape velocity $v_{\text {esc }}$ at that point. For $v>v_{\text {esc }}, F(\varepsilon)$ is

by definition zero. Furthermore, the formula shows that the DM velocity distribution at $R_{\odot}, F\left(\Psi_{\odot}-\frac{1}{2} v^{2}\right)$, depends only on the DM density distribution at $r>R_{\odot}$. Thus, halo density profiles sharing the same large $r$ behaviour will yield similar solutions for $F\left(\Psi_{\odot}-\frac{1}{2} v^{2}\right)[56]$. For this reason the NFW and the Moore profiles are for our purposes equivalent (see section 3.1).

The last step is to rewrite the velocity integral in the differential recoil rate $(2.1)$ in terms of $F\left(\Psi_{\odot}-\frac{1}{2} v^{2}\right)$, that is,

$$
\int_{v^{\prime}>v_{\min }^{\prime}} \mathrm{d}^{3} v^{\prime} \frac{f\left(\overrightarrow{v^{\prime}}(t)\right)}{v^{\prime}} \rightarrow 2 \pi \rho_{\odot}^{-1} \int_{v^{\prime}>v_{\min }^{\prime}} \mathrm{d} v^{\prime} v^{\prime} \int_{-1}^{1} \mathrm{~d} \alpha F\left(\Psi_{\odot}-\frac{1}{2} v^{2}\right),
$$

with

$$
\begin{aligned}
& v^{2}=\left|\overrightarrow{v^{\prime}}+\vec{v}_{\oplus}\right|^{2}=v^{\prime 2}+v_{\oplus}^{2}+2 v^{\prime} v_{\oplus} \alpha, \\
& v_{\oplus}=\left|\vec{v}_{\odot}+\vec{v}_{\oplus, \text { rot }}^{\prime \prime}\right|=v_{\odot}+v_{\oplus, \text { rot }}^{\prime \prime} \cos \gamma \cos \left[2 \pi\left(t-t_{0}\right) / T\right],
\end{aligned}
$$

where $\vec{v}_{\oplus}$ and $\vec{v}_{\odot}$ are, respectively, the Earth's and the sun's velocity in the Galactic frame, $\overrightarrow{v^{\prime \prime}} \oplus$,rot is the Earth's rotational velocity around the sun in the sun's rest frame, and $\gamma=60^{\circ}$ is the inclination of the Earth's rotation plane with respect the the Galactic 
plane. For our analysis we take $v_{\oplus, \text { rot }}^{\prime \prime}=29.8 \mathrm{~km} \mathrm{~s}^{-1}$, and $v_{\odot}=v_{0}+12 \mathrm{~km} \mathrm{~s}^{-1}[49,57]$, where

$$
\left.v_{0} \equiv \sqrt{-r \frac{\mathrm{d} \Psi}{\mathrm{d} r}}\right|_{r=R_{\odot}}
$$

is the circular velocity of the local standard of rest. In the time-dependent piece the period $T$ is one year, while $t_{0}$ corresponds to June 2 , the day on which $v_{\oplus}$ reaches its maximum.

Finally, we note that implicit in the recoil rate (2.1) are four astrophysical observables: the local DM density $\rho_{\odot}$, the Milky Way virial mass $M_{\text {vir }}$, the circular velocity of the local standard of rest $v_{0}$ defined in equation (3.12), and the local escape velocity,

$$
v_{\mathrm{esc}}=\left.\sqrt{2 \Psi}\right|_{r=R_{\odot}} .
$$

These can be independently constrained using observations of stellar and satellite kinematics. We discuss this point in more detail in section 4.7.

\section{Experiments and their likelihood functions}

The likelihood function $\mathcal{L}(X \mid \theta)$ denotes the probability of the data $X$ given some theoretical prediction $\theta$, and plays a central role in Bayesian inference. In this section we describe the likelihood function used for each experiment, as well as the modelling of potential systematics. An in-depth discussion of Bayesian methods is deferred to section 5. Table 1 summarises the free (MCMC) parameters of our analysis.

\subsection{CDMSSi}

The cryogenic CDMS experiment at the Soudan Underground Laboratory operates germanium and silicon solid-sate detectors. Two events were observed at 55 and $95 \mathrm{keVnr}$ in the silicon run (CDMSSi hereafter) on a $0.1 \mathrm{~kg}$ detector in an exposure of $65.8 \mathrm{~kg}$-days, compatible with an expected background of $B_{n}=3.6$ neutrons and $B_{e}=0.8 \pm 0.6$ electrons in the $5 \rightarrow 100 \mathrm{keVnr}$ detection window [17]. No quenching factor is required for the CDMS experiment, i.e., $q=1$. For details of the detector efficiency $\epsilon(q E)$ we refer the reader to, e.g., [58].

We model the corresponding likelihood function with a Poisson(2) distribution, $\ddagger$

$$
\ln \mathcal{L}_{\mathrm{CDMSSi}}(2 \mid S, B)=-S-B+2+2 \ln \left(\frac{S+B}{2}\right),
$$

where $S$ is the expected WIMP signal in the detection window, and $B=B_{n}+B_{e}$ the expected background. The likelihood function (4.1) is normalised such that $\ln \mathcal{L}=0$ if the sum of the expected signal and background matches exactly the number of observed events.

$\ddagger$ The notation Poisson $(n)$ denotes the Poisson distribution for $n$ observed events. 
Bayes and present DM direct detection

Table 1. MCMC parameters and priors for the WIMP parameter space and experimental systematics (nuisance parameters). All priors are uniform over the indicated range.

\begin{tabular}{lll}
\hline Experiment & MCMC parameter & Prior \\
\hline All & $\log \left(m_{\mathrm{DM}} / \mathrm{GeV}\right)$ & $0 \rightarrow 3$ \\
All & $\log \left(\sigma_{n}^{\mathrm{SI}} / \mathrm{cm}^{2}\right)$ & $-44(-46) \rightarrow-38$ \\
DAMA & $q_{\mathrm{Na}}$ & $0.2 \rightarrow 0.4$ \\
DAMA & $q_{\mathrm{I}}$ & $0.06 \rightarrow 0.1$ \\
Xenon100 & $m$ & $-0.01 \rightarrow 0.18$ \\
CoGeNT & $C$ & $0 \rightarrow 10 \mathrm{cpd} / \mathrm{kg} / \mathrm{keV}$ \\
CoGeNT & $\mathcal{E}_{0}$ & $0 \rightarrow 30 \mathrm{keV}$ \\
CoGeNT & $G_{n}$ & $0 \rightarrow 10 \mathrm{cpd} / \mathrm{kg} / \mathrm{keV}$ \\
CDMSGe(LE) & $a$ & $-0.60 \rightarrow-0.18$ \\
\hline
\end{tabular}

Since the expected background rate comes with an uncertainty -in this instance, $B=\bar{B} \pm \sigma_{B}=4.4 \pm 0.6$, it is useful to construct an effective likelihood function $\mathcal{L}^{\text {eff }}$ by marginalising over the background $B$,

$$
\mathcal{L}_{\mathrm{CDMSSi}}^{\mathrm{eff}}(2 \mid S)=\int_{0}^{\infty} \mathrm{d} B \mathcal{L}_{\mathrm{CDMSSi}}(2 \mid S, B) p(B),
$$

where

$$
p(B)=\frac{1}{\sqrt{2 \pi \sigma_{B}^{2}}} \exp \left[-\frac{(B-\bar{B})^{2}}{2 \sigma_{B}^{2}}\right]
$$

is the probability density function of $B$ (modelled as a Gaussian distribution). In the small $\sigma_{B} / \bar{B}$ limit, the resulting effective likelihood has the form,

$$
\ln \mathcal{L}_{\mathrm{CDMSSi}}^{\mathrm{eff}}=-S-\bar{B}+\frac{\sigma_{B}^{2}}{2}+2+\ln \left[\frac{\sigma_{B}^{2}+\left(S+\bar{B}-\sigma_{B}^{2}\right)^{2}}{4}\right],
$$

which we use in our inference analysis.§

\section{2. $C D M S G e$}

For their germanium run, the CDMS-II (CDMSGe hereafter) reported two events at 12.3 and $15.5 \mathrm{keVnr}$ in the $10 \rightarrow 100 \mathrm{keVnr}$ window in a total exposure of $612 \mathrm{~kg}$-days [18]. The total expected background in the same time frame is $B=0.8 \pm 0.1 \pm 0.2$. For our analysis, however, we adopt the fitting formula for the differential background rate provided by [24],

$$
\frac{\mathrm{d} N_{B}}{\mathrm{~d} E}=\left[-0.00295+0.463\left(\frac{\mathrm{keVnr}}{E}\right)\right] /(612 \mathrm{~kg} \text { days }),
$$

$\S$ We adopt the small $\sigma_{B} / \bar{B}$ limit results whenever $\bar{B} \gtrsim 3 \sigma_{B}$. 
where the rate has been normalised to $\bar{B}=0.8$ events over the detection window in an exposure of $612 \mathrm{~kg}$-days. Exploiting this spectral information, we model the likelihood function as a product of two Poisson(1) distributions (for those energies with one event each) and a series of Poisson(0) distributions (for those energies with no events) [59], that is,

$$
\ln \mathcal{L}_{\mathrm{CDMSGe}}=-S-B+2+\sum_{i=1,2} \ln \left(\frac{\mathrm{dR}}{\mathrm{dE}_{\mathrm{i}}}+\frac{B}{\bar{B}} \frac{\mathrm{d} N_{B}}{\mathrm{~d} E_{i}}\right)+C_{\text {norm }},
$$

where $S$ and $B$ are, respectively, the total expected signal and background in the detection window, $E_{1,2}=12.3,15.5 \mathrm{keVnr}$, and $C_{\text {norm }}=\sum_{i=1,2} \ln \left[M_{\operatorname{det}} T \epsilon\left(q E_{i}\right)\right]$ is a normalisation factor following from the normalisation of the individual Poisson(1) and Poisson(0) distributions. See discussion after equation (4.1).

Marginalising over the total background $B$ (but not the spectral shape) in the manner of equations (4.2) and (4.3), we find in the small $\sigma_{B} / \bar{B}$ limit an effective likelihood

$$
\begin{aligned}
\ln \mathcal{L}_{\mathrm{CDMSGe}}^{\mathrm{eff}}= & -S-\bar{B}+\frac{\sigma_{B}^{2}}{2}+2+C_{\mathrm{norm}}+ \\
& \ln \left[\prod_{i=1,2}\left(\frac{\mathrm{d} R}{\mathrm{~d} E_{i}}+\frac{\bar{B}-\sigma_{B}^{2}}{\bar{B}} \frac{\mathrm{d} N_{B}}{\mathrm{~d} E_{i}}\right)+\sigma_{B}^{2} \prod_{i=1,2} \frac{1}{\bar{B}} \frac{\mathrm{d} N_{B}}{\mathrm{~d} E_{i}}\right] .
\end{aligned}
$$

We include in the analysis also null results from three previous searches with the CDMS germanium detector, with exposures of $34 \mathrm{~kg}$-days [17], $19.4 \mathrm{~kg}$-days [60], and $397.8 \mathrm{~kg}$ days [61], bringing the total exposure to $1063.2 \mathrm{~kg}$-days. The expected background and its uncertainty are scaled correspondingly to $\bar{B}=1.39$ and $\sigma_{B}=0.38$ respectively. We model the detector efficiency $\epsilon(q E)$ after [24].

Low energy CDMS The CDMS collaboration has recently re-analysed their germanium data - both on their own, and in combination with data from the silicon detectors - with a lower energy threshold $[62,63]$, thereby increasing the experiment's sensitivity to light WIMPs. In reference [62], data from 8 germanium detectors (CDMSGe(LE) hereafter) were re-analysed using a threshold of $2 \mathrm{keVnr}$, compared to $10 \mathrm{keVnr}$ in the standard analysis. For each detector, the collaboration provides the event energies and the raw exposure. After summing up all contributions and applying the efficiency cuts one finds a total of 427 counts for $214 \mathrm{~kg}$-days, distributed in the energy range $2 \rightarrow 100 \mathrm{keVnr}$. In our analysis, we bin the data in such a way that 16 bins are contained in the energy range $2 \rightarrow 10 \mathrm{keVnr}$, and 9 in $10 \rightarrow 100 \mathrm{keVnr}$.

A lower energy threshold, unfortunately, is traded at the cost of an increased acceptance of background events, because at these low energies the ability of the experiment to discriminate between nuclear and electron recoils degrades and the ionization signal becomes dominated by noise. Indeed, while the background due to surface events, "zero-charge" events, and leakage events are reasonably well known at energies > $5 \mathrm{keVnr}$, between $2 \mathrm{keVnr}$ and $5 \mathrm{keVnr}$ the CDMS collaboration has to rely on an extrapolation to model these events in their analysis, as described in figure 1 
of [62]. Another potential issue is the calibration of the recoil energy near threshold, since the ionisation signal is missing.

Given these considerations, we model the differential background rate in our analysis as

$$
\mathrm{m}_{\mathrm{B}}(E)= \begin{cases}\overline{\mathrm{m}}_{\mathrm{B}}(E), & E \geq 5 \mathrm{keVnr}, \\ 0.1 \times 10^{a[(E / \mathrm{keVnr})-5],} & 2<E / \mathrm{keVnr}<5,\end{cases}
$$

where $\overline{\mathrm{m}}_{\mathrm{B}}(E)$ corresponds to the black curve in figure 1 of [62], which, for energies above $5 \mathrm{keVnr}$, we regard as reliable and free of systematics. For energies below $5 \mathrm{keVnr}$ we use an extrapolation function, but allow the slope $a$ to vary subject to a Gaussian constraint

$$
\ln \mathcal{L}_{m_{\mathrm{B}}}=-\frac{(a-\bar{a})^{2}}{2 \sigma_{a}^{2}},
$$

where the "best-fit" $\bar{a}=-0.36$ reproduces the black curve in figure 1 of [62], and $\sigma_{a}=0.2 \bar{a}$, chosen based on the error bars in the same figure at $5 \mathrm{keV}$.

The expected signal rate in the $i$ th energy bin is then a sum of the DM signal and the background rate,

$$
s_{i}=\frac{1}{\Delta E_{i}} \int_{E_{i}-\Delta E_{i} / 2}^{E_{i}+\Delta E_{i} / 2} \mathrm{~d} E\left[\frac{\mathrm{d} R}{\mathrm{~d} E}+\mathrm{m}_{\mathrm{B}}(E)\right],
$$

where $\Delta E_{i}$. The likelihood function is given by

$$
\ln \mathcal{L}_{\mathrm{CDMSGe}(\mathrm{LE})}=-\sum_{i=1}^{N_{\text {bin }}} \frac{\left(s_{i}-\bar{s}_{i}^{\mathrm{obs}}\right)^{2}}{2 \sigma_{i}^{2}}+\ln \mathcal{L}_{m_{\mathrm{B}}} .
$$

where $\bar{s}_{i}^{\text {obs }}$ is the observed rate in the $i$ th bin, and $\sigma_{i}$ is the associated error.

We do not consider the re-analysis of the combined data on the germanium and the silicon towers presented in [63], because the lack of knowledge about the low-energy background makes it difficult for us to model the likelihood function.

\section{3. $\mathrm{CoGeNT}$}

The CoGeNT experiment, an ultra low-noise (and hence low-threshold: 0.4 keVee) germanium cryogenic detector running at the Soudan Mine, found in a total exposure of $18.48 \mathrm{~kg}$-days an excess at low energies that cannot be attributed to known background sources [2]. Using the energy binning in figure 3 of [2] in the $0.4 \rightarrow 3.2$ keVee energy range, we model the likelihood function as a sum of Poisson $\left(X_{i}\right)$ distributions,

$$
\ln \mathcal{L}_{\mathrm{CG}}=\sum_{i=1}^{56}\left[-s_{i}-b_{i}-r_{i}+X_{i}+X_{i} \ln \left(\frac{s_{i}+b_{i}+r_{i}}{X_{i}}\right)\right],
$$

where $X_{i}$ is the number of events observed in the $i$ th energy bin, $s_{i}$ is the expected signal computed from equation (2.4) with $\mathcal{E}_{1}$ and $\mathcal{E}_{2}$ corresponding respectively to the lower and upper energy limits of the bin concerned, and $b_{i}$ and $r_{i}$ are two background components. 
For the first component $b_{i}$, we model the differential rate as an exponentially decaying function,

$$
\frac{\mathrm{d} N_{b}}{\mathrm{~d} \mathcal{E}}=C \exp \left(-\mathcal{E} / \mathcal{E}_{0}\right),
$$

where $C$ and $\mathcal{E}_{0}$ are two free parameters. The second component $r_{i}$ denotes events due to two radiation peaks from ${ }^{65} \mathrm{Zn}$ and ${ }^{68} \mathrm{Ge}$ decays, whose differential rates are modelled as Gaussians centered on the energies $\mathcal{E}_{\mathrm{Zn}}=1.1 \mathrm{keVee}$ and $\mathcal{E}_{\mathrm{Ge}}=1.29 \mathrm{keVee}$, with a common standard deviation fixed by the energy resolution of the detector $\Delta \mathcal{E}$, i.e.,

$$
\frac{\mathrm{d} N_{\mathrm{Zn}}}{\mathrm{d} \mathcal{E}}=G_{n, \mathrm{Zn}} \exp \left(-\frac{\left(\mathcal{E}-\mathcal{E}_{1}\right)^{2}}{2 \Delta \mathcal{E}^{2}}\right)
$$

and similarly for $d N_{\mathrm{Ge}} / d E$. We fix the ratio of the two peak heights to $G_{n, \mathrm{Zn}} / G_{n, \mathrm{Ge}}=0.7$, and vary only $G_{n} \equiv G_{n, \mathrm{Ge}}$. For details about cosmogenic backgrounds we refer to [64]. The energy resolution is given by $\Delta \mathcal{E} / \mathrm{eVee}=\sqrt{\sigma_{n}^{2}+2.96 F(\mathcal{E} / \mathrm{eVee})}$, with $\sigma_{n}=69.4$ and $F=0.29$ [2], while the energy-dependent quenching factor is taken to be $q=2 /[1+\sqrt{1+15.55(\mathrm{keVee} / \mathcal{E})}]$, following $[24]$.

Because the background model parameters $C, \mathcal{E}_{0}$ and $G_{n}$ enter into the likelihood function (4.12) in a nontrivial fashion, analytical marginalisation in the manner of equation (4.2) is cumbersome if not impossible. We therefore treat these parameters as MCMC parameters.

\subsection{Xenon}

Xenon is a two phase (liquid/gas) xenon experiment running at Laboratori Nazionali del Gran Sasso (LNGS). A nuclear recoil from particle scattering is inferred from the simultaneous measurements of scintillation light and ionisation electrons, together with the arrival direction. The amount of nuclear recoil energy going into the primary scintillation signal is expressed in terms of the number of photoelectrons (PE) produced $S_{1}$, which is related to the nuclear recoil energy $E$ through the relation

$$
S_{1}(E)=\mathrm{L}_{\mathrm{eff}}(E) \mathrm{L}_{y} E \frac{S_{\mathrm{nr}}}{S_{\mathrm{ee}}},
$$

where $\mathrm{L}_{\mathrm{eff}}(E)$ is the energy-dependent scintillation efficiency, $\mathrm{L}_{y}=2.2 \mathrm{PE} / \mathrm{keVee}$ the scintillation efficiency of nuclear recoils relative to that of the 122 keVee $\gamma$-rays at zero field, and the quantities $S_{\mathrm{nr}, \mathrm{ee}}=0.95,0.58$ denote respectively the electric field scintillation quenching factors for nuclear and electron recoils.

Between 2006 and 2007, the Xenon10 collaboration found 13 events for an expected background of 7 events in an exposure of $316.4 \mathrm{~kg}$-days in the $2.0 \rightarrow 75.0 \mathrm{keVnr}$ window [20,65]. The Xenon100 experiment recently released an analysis of 100.9 live days of data acquired in 2010, which found three candidate events for an expected background of $B=1.8 \pm 0.6$ in an exposure of $1481 \mathrm{~kg}$ days. [19]. Because of this large exposure, we consider in this work only the results of Xenon100 from the aforementioned data release, although the analysis techniques can easily be generalised for used with Xenon10. 
As in the case of CDMSGe we include both the total rate and spectral information in the likelihood,

$$
\ln \mathcal{L}_{\text {Events }}=-S-B+3+\sum_{i=1}^{3} \ln \left(\left.\frac{\mathrm{d} R}{\mathrm{~d} S_{1}}\right|_{i}+\left.\frac{B}{\bar{B}} \frac{\mathrm{d} N_{B}}{\mathrm{~d} S_{1}}\right|_{i}\right)+C_{\text {norm }},
$$

where three events are at 8,20 , and $23 \mathrm{PE}$, respectively, and $C_{\text {norm }}=\sum_{i=1,2,3} \ln \left(M_{\text {det }} T\right)$ is a constant normalisation factor. The background has been shown to consist mainly of electron recoils, with a flat distribution in energy over the experimental range according to measurements and Monte Carlo simulations [66]. We therefore model the differential background as $\mathrm{d} N_{B} / \mathrm{d} S_{1}=0.069 /(1481 \mathrm{~kg}$ days $)$, normalised to $\bar{B}=1.8$ in the detection window of $4 \rightarrow 30 \mathrm{PE}$ in $1481 \mathrm{~kg}$ days.

The expected WIMP signal $S$ is computed as follows. Firstly, we note that the actual conversion between the nuclear recoil energy and the number of PEs produced is not deterministic. Given some recoil energy $E$, the number of PEs produced $S_{1}$ is subject to Poisson fluctuations, with equation (4.15) expressing only the expectation value $\bar{S}_{1}$. In physical terms this means recoils below the nominal energy threshold have a finite probability of leaking into the detection window of the experiment, and this effect is important for the detection of light WIMPs. The expected number of WIMP events as a function of the (discrete) number of PEs generated can be written as

$$
\frac{\mathrm{d} R}{\mathrm{~d} S_{1}}=\int_{0}^{\infty} \mathrm{d} E \frac{\mathrm{d} R}{\mathrm{~d} E} \times P\left(S_{1} \mid \bar{S}_{1}(E)\right)
$$

where $P\left(S_{1} \mid \bar{S}_{1}(E)\right)$ denotes a Poisson $(n)$ distribution with expectation $\bar{S}_{1}(E)$. Summing over all PE counts, the total number of events expected in the detector is

$$
S=M_{\mathrm{det}} T \sum_{n=\mathrm{PE}_{\min }}^{\mathrm{PE}_{\max }} \frac{\mathrm{d} R}{\mathrm{~d} S_{1}}
$$

where for Xenon100, $\mathrm{PE}_{\min }=4$ and $\mathrm{PE}_{\max }=30$.

It remains to specify the energy-dependent scintillation efficiency $\mathrm{L}_{\text {eff }}(E)$. In this work we use,

$$
\mathrm{L}_{\mathrm{eff}}(E)= \begin{cases}\overline{\mathrm{L}}_{\mathrm{eff}}(E), & E \geq 3 \mathrm{keVnr}, \\ \max \{m[\ln (E / \mathrm{keVnr})-\ln 3]+0.09, \quad 0\}, & 1<E / \mathrm{keVnr}<3 .\end{cases}
$$

Here, $\overline{\mathrm{L}}_{\text {eff }}(E)$ corresponds to the best-fit in figure 1 of [19], which, at $E \geq 3 \mathrm{keVnr}$, is well-constrained by direct measurements. No direct measurements exists at $1<$ $E /$ keVnr $<3$, and the "best-fit" provided by the Xenon100 collaboration is merely an extrapolation. We therefore treat the extrapolation slope $m$ as a variable, MCMC parameter, subject to a Gaussian constraint of

$$
\ln \mathcal{L}_{\mathrm{L}_{\mathrm{eff}}}=-\frac{(m-\bar{m})^{2}}{2 \sigma_{m}^{2}}
$$

where $\bar{m} \equiv 0.082$ reproduces the "best-fit" of [19] in the $1<E / \mathrm{keVnr}<3$ region, and $\sigma_{m}=0.04$, chosen so that the $2 \sigma$ region coincides approximately with the light blue 
band. We further restrict $m$ to lie within the range $[-0.01,0.18]$, so that $L_{\text {eff }}(E)$ never exceeds 0.1 at $E=1 \mathrm{keVnr}$, or drops to zero at energies above $2 \mathrm{keVnr}$.

We note that a somewhat different parameterisation for the uncertainty in $\mathrm{L}_{\text {eff }}(E)$ was adopted in [66], which also accounts for errors in the direct measurements of $\mathrm{L}_{\mathrm{eff}}(E)$ at $E \geq 3 \mathrm{keVnr}$. Nonetheless we choose the parameterisation (4.19) because it highlights the role of $\mathrm{L}_{\mathrm{eff}}(E)$ in the low energy region. Indeed, from equation (4.17), we see that the main uncertainty in the expected event rate at energies close to the threshold comes from Poisson fluctuations in the number of PEs produced, which in turn depend sensitively on the unknown slope of $\mathrm{L}_{\text {eff }}(E)$ at $E<3 \mathrm{keVnr}$ via equation (4.15). The small errors around the best-fit $\mathrm{L}_{\mathrm{eff}}(E)$ at $E>3 \mathrm{keVnr}$, on the other hand, has little impact on the physics close to the threshold and hence also the exclusion power of the experiment for light WIMPs. Note that our likelihood function (4.16) automatically takes into account uncertainties in $L_{\text {eff }}(E)$ in both the total number of signal events and their energy dependence, in contrast to the approach of [66], which explicitly assumes the (normalised) signal energy spectrum to be independent of these uncertainties.

Thus, the full likelihood function describing the Xenon100 experiment is

$$
\ln \mathcal{L}_{\text {Xenon }}=\ln \mathcal{L}_{\text {Events }}+\ln \mathcal{L}_{\mathrm{L}_{\mathrm{eff}}}
$$

which we further marginalise numerically over the background events $B$ (in the $\ln \mathcal{L}_{\text {Events }}$ term) as per equations (4.2) and (4.3), yielding an effective likelihood that depends only on $m_{\mathrm{DM}}, \sigma_{n}^{\mathrm{SI}}$, and the systematics nuisance parameter $m$.

The Xenon10 collaboration recently published a low-energy analysis based on the ionisation signal (the $S_{2}$ signal) [67]. This alternative approach removes the dependence of the result on the uncertainties of the scintillation efficiency, thereby lowering the detector threshold significantly down to $1 \mathrm{keV}$. However, without a reliable parameterisation of the estimated background, it is not possible to construct a meaningful likelihood function for our Bayesian analysis. Since the Xenon10 collaboration does not provide the necessary information, we refrain from using their data in this work.

\subsection{DAMA}

The DAMA/Libra [1,68] experiment at LNGS uses $\mathrm{NaI}(\mathrm{Tl})$ crystal radio-pure scintillators as targets. The signature of WIMP interactions consists of an annual modulation of the signal due to the motion of the Earth through the Galactic halo as discussed in section 3.2. For a cumulative exposure of 1.17 ton-year, the collaboration reported a positive detection at $8.9 \sigma$ significance.

For isotropic WIMP velocity distributions such as those considered in this work, the expected modulation signal averaged over an observed energy interval $\left[\mathcal{E}_{1}, \mathcal{E}_{2}\right]$ is given by

$$
s=\frac{1}{\mathcal{E}_{2}-\mathcal{E}_{1}} \sum_{X=\mathrm{Na}, \mathrm{I}} w_{X} \int_{\mathcal{E}_{1} / q_{X}}^{\mathcal{E}_{2} / q_{X}} \mathrm{~d} E \frac{1}{2}\left[\frac{\mathrm{d} R_{X}}{\mathrm{~d} E}(\text { June } 2)-\frac{\mathrm{d} R_{X}}{\mathrm{~d} E}(\text { Dec } 2)\right],
$$


Table 2. Additional MCMC parameters and (uniform) priors related to the modelling of the WIMP velocity distribution.

\begin{tabular}{lll}
\hline Density profile & MCMC parameter & Prior \\
\hline All & $M_{\text {vir }}$ & $1 \rightarrow 5 \times 10^{12} M_{\odot}$ \\
NFW, Einasto & $c_{\text {vir }}$ & $5 \rightarrow 20[74]$ \\
Cored isothermal, Burkert & $c_{\text {vir }}$ & $50 \rightarrow 200[75]$ \\
\hline
\end{tabular}

where $w_{X} \equiv M_{X} /\left(M_{\mathrm{Na}}+M_{\mathrm{I}}\right)$, and we have explicitly ignored the small contribution from channelling [69]. The likelihood follows a Gaussian distribution,

$$
\ln \mathcal{L}_{\text {DAMA }}=-\sum_{i=1}^{N_{\text {bin }}} \frac{\left(s_{i}-\bar{s}_{i}^{\text {obs }}\right)^{2}}{2 \sigma_{i}^{2}},
$$

where $s_{i}$ and $\bar{s}_{i}^{\text {obs }}$ are the theoretical and the mean observed modulation respectively in the $i$ th energy bin, $\sigma_{i}$ is the associated uncertainty in the observed signal, and we use in this analysis the 36-bin data from figure 9 of [68]. The quenching factors $q_{\mathrm{Na}}$ and $q_{\mathrm{I}}$ are taken to be free parameters in our analysis, which we vary, respectively, over a range representative of the diverse measured values found in the literature [70-73].

\subsection{Other experiments}

Even though we will not consider their results in our analysis, let us also mention the following direct detection experiments.

The CRESST collaboration has found 32 events on oxygen given an expected background of $8.7 \pm 1.4$ [76]. If interpreted as a WIMP signal, this would point to a low mass WIMP with a coherent cross-section in the ballpark of the regions preferred by DAMA and CoGeNT data [7].

The Edelweiss collaboration recently published the final analysis for their second run, reporting 5 events for an expected background of 3, 4 of which are close to the threshold [21]. An exclusion bound was set, which, because of the smaller exposure of the experiment, is not competitive with those derived from other experiments considered in this work. A recent combined analysis of Edelweiss and CDMS has improved the sensitivity: a tighter exclusion bound for DM masses above $200 \mathrm{GeV}$ was found, relative to the limits obtained by the individual experiment alone [77]. However, this new limit is still less constraining than that derived from the Xenon100 experiment.

\subsection{Astrophysics}

In addition to the WIMP mass, cross-section, and the nuisance parameters of the direct search experiments, two further free parameters are used to characterise the WIMP velocity distribution: the virial mass of the DM halo, and its concentration (see 
Table 3. Astrophysical constraints on the DM halo profile and the WIMP velocity distribution.

\begin{tabular}{ll}
\hline Observable & Constraint \\
\hline Local standard of rest & $v_{0}^{\text {obs }}=230 \pm 24.4 \mathrm{~km} \mathrm{~s}^{-1}[78,79]$ \\
Escape velocity & $v_{\text {esc }}^{\text {obs }}=544 \pm 39 \mathrm{~km} \mathrm{~s}^{-1}[80,81]$ \\
Local DM density & $\rho_{\odot}^{\text {obs }}=0.4 \pm 0.2 \mathrm{GeV} \mathrm{cm}^{-3}[82,83]$ \\
Virial mass & $M_{\mathrm{vir}}^{\text {obs }}=2.7 \pm 0.3 \times 10^{12} M_{\odot}[84,85]$ \\
\hline
\end{tabular}

table 2). These additional parameters are, however, also constrained by astrophysical observations. For this reason, we define a likelihood function for the astrophysics,

$$
\ln \mathcal{L}_{\text {Astro }}=-\frac{\left(v_{0}-\bar{v}_{0}^{\mathrm{obs}}\right)^{2}}{2 \sigma_{v_{0}}^{2}}-\frac{\left(v_{\mathrm{esc}}-\bar{v}_{\mathrm{esc}}^{\mathrm{obs}}\right)^{2}}{2 \sigma_{v_{\mathrm{esc}}}^{2}}-\frac{\left(\rho_{\odot}-\bar{\rho}_{\odot}^{\mathrm{obs}}\right)^{2}}{2 \sigma_{\rho_{\odot}}^{2}}-\frac{\left(M_{\mathrm{vir}}-\bar{M}_{\mathrm{vir}}^{\mathrm{obs}}\right)^{2}}{2 \sigma_{M_{\mathrm{vir}}}^{2}},
$$

where the measured values of the various astrophysical observables (see section 3.2 for their definitions) and their uncertainties are given in table 3. Note that none of the constraints in table 3 assumes a specific parameterisation of the halo profile, which allows us to apply them to all halo models we are considering here without running the risk of double-fitting.

\section{Statistical inference}

Having specified a theoretical model with free parameters $\theta$ in sections 2 and 3 , and defined the likelihood functions $\mathcal{L}(X \mid \theta)$ in section 4 , one final step remains to be taken in the analysis of the data $X$ : the inference of the posterior probability density as a function of the parameters, $\mathcal{P}(\theta \mid X)$. The posterior pdf represents our state of knowledge about the parameters after taking into account the information contained in the data, and has an intuitive and straightforward interpretation in that $\int_{V} \mathcal{P}(\theta \mid X) \mathrm{d} \theta$ is the probability that the true value of $\theta$ lies in the volume $V$. Given a likelihood function, the posterior pdf can be constructed by invoking Bayes' theorem,

$$
\mathcal{P}(\theta \mid X) \mathrm{d} \theta \propto \mathcal{L}(X \mid \theta) \cdot \pi(\theta) \mathrm{d} \theta,
$$

but the construction requires us to specify $\pi(\theta)$, the probability density on the parameter space $\theta$ prior to observing the data $X$. Since this prior pdf is independent of the data, it needs to be chosen according to one's theoretical prejudice, and is thus inherently subjective.

In the often encountered situation in which no unique theoretically motivated prior pdf can be derived, one may wish to use one which does not favour any parameter region 
in particular. A common choice in this case is the top-hat, or uniform, prior

$$
\pi_{\text {flat }}(\theta) \mathrm{d} \theta \propto\left\{\begin{array}{cl}
\mathrm{d} \theta, & \text { if } \theta_{\min } \leq \theta \leq \theta_{\max }, \\
0, & \text { otherwise }
\end{array}\right.
$$

if the general order of magnitude of the parameter is known. Here, the limits $\theta_{\min }$ and $\theta_{\max }$ should be chosen such that they are well beyond the parameter region of interest. If even the order of magnitude is unknown, one may want to choose a uniform prior in $\log \theta$ space instead,

$$
\pi_{\log }(\log \theta) \mathrm{d} \log \theta=\left\{\begin{array}{cl}
\mathrm{d} \log \theta, & \text { if } \theta_{\min } \leq \theta \leq \theta_{\max } \\
0, & \text { otherwise }
\end{array}\right.
$$

which is equivalent to a $\mathrm{d} \theta / \theta$ prior in $\theta$ space. Note that because the volume element $\mathrm{d} \theta$ is in general not invariant under a parameter transformation $f: \theta \rightarrow \theta^{\prime}$, a uniform prior pdf on $\theta$ does not yield the same probabilities as a uniform prior pdf on $\theta^{\prime}$ unless the mapping $f$ is linear. The same is also true for the posterior probabilities, i.e., $\mathcal{P}(\theta \mid X) \mathrm{d} \theta \neq \mathcal{P}\left(\theta^{\prime} \mid X\right) \mathrm{d} \theta^{\prime}$ in general.

While the posterior pdf technically contains all the necessary information for the interpretation of the data, the fact that it is a function in the $N$-dimensional space of parameters makes it difficult to visualise if $N>2$. Fortunately, by virtue of being a probability density, its dimensionality can be easily reduced by integrating out less interesting (nuisance) parameter directions $\psi_{i}$, yielding an $n$-dimensional marginal posterior pdf,

$$
\mathcal{P}_{\text {mar }}\left(\theta_{1}, \ldots, \theta_{n} \mid X\right) \propto \int \mathrm{d} \psi_{1} \ldots \mathrm{d} \psi_{m} \mathcal{P}\left(\theta_{1}, \ldots, \theta_{n}, \psi_{1} \ldots, \psi_{m} \mid X\right),
$$

which is more amenable to visual presentation if $n=1,2$, and can be used to construct constraints on the remaining parameters.

A complementary approach to the marginalisation is to project the likelihood function $\mathcal{L}(X \mid \theta)$ onto the $n$-dimensional subspace by maximising along the nuisance directions, i.e,

$$
\mathcal{L}_{\text {prof }}\left(X \mid \theta_{1}, \ldots, \theta_{n}\right) \propto \max _{\psi_{1} \ldots \psi_{m}} \mathcal{L}\left(X \mid \theta_{1}, \ldots, \theta_{n}, \psi_{1} \ldots, \psi_{m}\right) .
$$

Maximisation is not a Bayesian procedure, and the resulting profile likelihood cannot be interpreted as a probability density function. However, because $\mathcal{L}_{\text {prof }}$ is by construction insensitive to our choice of priors and associated volume effects, it can be a useful means to assess if the inference has been significantly affected by our choice of nuisance parameterisation.\|

\subsection{Priors}

The main parameters of interest in this work are $m_{\mathrm{DM}}$ and $\sigma_{n}^{\mathrm{SI}}$. These are accompanied by a set of astrophysical and experiment-specific systematic nuisance parameters, as discussed in section 4 .

$\|$ We always normalise the (marginal) posterior pdf and profile likelihood so that $\max \left(\mathcal{P}_{\operatorname{mar}}\right)=$ $\max \left(\mathcal{L}_{\text {prof }}\right)=1$. 
When it comes to specifying prior pdfs for $m_{\mathrm{DM}}$ and $\sigma_{n}^{\mathrm{SI}}$, we have very little guidance from theory without resorting to specific dark matter models. As long as the dark matter is cold, massive and weakly enough interacting, pretty much all combinations of values are a priori allowed. It thus appears reasonable to impose uniform priors on both $\log m_{\mathrm{DM}}$ and $\log \sigma_{n}^{\mathrm{SI}}$. With the assumption that the dark matter particle is a WIMP, we can at least roughly confine our prior region. For definiteness, we take $\log \left(m_{\mathrm{DM}} / \mathrm{GeV}\right)$ to lie in the range $0 \rightarrow 3$ and allow $\log \left(\sigma_{n}^{\mathrm{SI}} / \mathrm{cm}^{2}\right)$ to vary between $-46 \rightarrow-38$, as reported in table 1 .

Interestingly, the choice of prior boundaries on $m_{\mathrm{DM}}$ and $\sigma_{n}^{\mathrm{SI}}$ also translates directly to how likely we deem the direct detection experiments to actually make a positive detection. Consider for instance the loss of detection sensitivity for large DM masses (due to the large mass splitting between the DM particle and the nucleus), or for very light WIMPs (because of the energy threshold): the larger the prior-space in the

$\left\{m_{\mathrm{DM}}, \sigma_{n}^{\mathrm{SI}}\right\}$-plane, the smaller the relative fraction that the experiments will be able to constrain, and the smaller the subjective prior probability for them to see something.

Our priors for the astrophysical parameters $M_{\text {vir }}$ and $c_{\text {vir }}$ are listed in table 2 . The ranges for the concentration parameters are inferred from simulations [74] for the NFW and Einasto profiles, and from fits the rotation curves of galaxies for Cored isothermal and Burkert profiles [75]. Note that since $M_{\text {vir }}$ is well-constrained by measurements (see table 3), the likelihood at the prior boundaries is negligible, and the inferred posterior pdf will be independent of our exact choice of prior boundaries for this parameter.

\subsection{Numerical implementation and construction of parameter constraints}

We employ a modified version of the public MCMC code CosmoMC [86,87], which uses the Metropolis-Hastings algorithm [88,89] to sample the posterior over the full parameter space. The resulting chains are analysed with an adapted version of the accompanying package GetDist, supplemented with matlab scripts from the package SuperBayes [29,90]. One- or two-dimensional marginal posterior pdfs are obtained from the chains by dividing the relevant parameter subspace into bins and counting the number of samples per bin. An $x \%$ credible interval or region containing $x \%$ of the total volume of $\mathcal{P}_{\text {mar }}$ is then constructed by demanding that $\mathcal{P}_{\text {mar }}$ at any point inside the region be larger than at any point outside. In the one-dimensional case, a credible interval thus constructed corresponds to the Minimal Credible Interval of [91]. Our profile likelihoods are also computed using CosmoMC, but with a 100-fold increase in the number of likelihood evaluations, so as to ensure that the tails of the distributions are well sampled and the true global maximum located.

Provided the data are sufficiently constraining - that is, if the prior pdf is nearly constant and, under a parameter transformation $f: \theta \rightarrow \theta^{\prime}$, the mapping $f$ is almost linear over the parameter region where the likelihood is large - the marginal posterior typically exhibits very little dependence on the choice of prior. For data that can only provide an upper or a lower bound on a parameter (or no bound at all) however, the 
properties of the inferred posterior and the boundaries of credible regions can vary significantly with the choice of prior as well as its limits $\theta_{\min }$ and $\theta_{\max }$, making an objective interpretation of the results rather difficult. As we shall see in the next section, this is in fact the case for the inference of credible regions in the $\left\{m_{\mathrm{DM}}, \sigma_{n}^{\mathrm{SI}}\right\}$-plane from Xenon100, CDMSSi and CDMSGe.

In these cases, in addition to computing credible intervals from the fractional volume of the marginal posterior in the $\left\{m_{\mathrm{DM}}, \sigma_{n}^{\mathrm{SI}}\right\}$-subspace $\mathcal{P}_{\operatorname{mar}}\left(m_{\mathrm{DM}}, \sigma_{n}^{\mathrm{SI}} \mid X\right)$, we also construct intervals based on the volume of the marginal posterior in $S$-space $\mathcal{P}_{\text {mar }}(S \mid X)$, where $S$ is the expected WIMP signal, using a uniform prior on $S$ with a lower boundary at zero [92]. An $x \%$ upper bound thus constructed has a welldefined Bayesian interpretation that the probability of $S \leq S_{x}$ is $x \%$. The limit $S_{x}$ is then mapped onto the $\left\{m_{\mathrm{DM}}, \sigma_{n}^{\mathrm{SI}}\right\}$-plane by identifying those combinations of $m_{\mathrm{DM}}$ and $\sigma_{n}^{\mathrm{SI}}$ with $\mathcal{P}_{\text {mar }}\left(m_{\mathrm{DM}}, \sigma_{n}^{\mathrm{SI}} \mid X\right)=\mathcal{P}_{\text {mar }}\left(S_{x} \mid X\right)$. An $x \%$ contour computed in this manner has the property of being independent of our choice of prior boundaries for $m_{\mathrm{DM}}$ and $\sigma_{n}^{\mathrm{SI}}$. Its drawback, however, is that it has no well-defined probabilistic interpretation in $\left\{m_{\mathrm{DM}}, \sigma_{n}^{\mathrm{SI}}\right\}$-space. $\uparrow$ To distinguish these $S$-based credible intervals from the conventional ones based on the volume of $\mathcal{P}_{\text {mar }}\left(m_{\mathrm{DM}}, \sigma_{n}^{\mathrm{SI}} \mid X\right)$, we label them with a subscript " $S$ ", e.g., $90_{S} \%$.

\section{Results}

We present our inference results in three parts. In section 6.1 we discuss the preferred parameter regions in $m_{\mathrm{DM}}$ and $\sigma_{n}^{\mathrm{SI}}$ for each experiment assuming the SMH (i.e., fixed astrophysics), after marginalising over the nuisance parameters of the experiments. In section 6.2 we vary in addition the WIMP velocity distribution in accordance with the DM density profile defined in section 3, and consider the effect of uncertainties in the astrophysics parameters on the inferred WIMP parameter values. Finally in section 6.3 we entertain the possibility of a combined analysis of the DAMA and the CoGeNT data.

\subsection{Standard model halo}

$D A M A$ Figure 1 shows our inference for the DAMA 36-bin data. The top panel shows the $2 \mathrm{D}$ marginal posterior pdf and the profile likelihood in the $\left\{m_{\mathrm{DM}}, \sigma_{n}^{\mathrm{SI}}\right\}$ subspace, where the two quenching factors $q_{\mathrm{Na}}$ and $q_{\mathrm{I}}$ have been integrated and profiled out respectively. Both approaches single out two preferred islands of parameter space in $\left\{m_{\mathrm{DM}}, \sigma_{n}^{\mathrm{SI}}\right\}$. Moreover, the colour coding indicates that $\mathcal{P}_{\text {mar }}\left(m_{\mathrm{DM}}, \sigma_{n}^{\mathrm{SI}}\right)$ and

I Clearly, the definition of the $S$-based bound and its associated probabilistic interpretation are contingent to our choice of a uniform prior on $S$; Had we chosen a different prior a different set of limits would have resulted. Our motivation for using a uniform prior stems from the observation that, for Poisson statistics, a Bayesian limit on $S$ constructed in the manner described turns out to have a well-defined interpretation in classical statistics, albeit a coincidental one [93]. This means the $S$-based bounds in this work can also be viewed as examples of the hybrid Bayesian/classical approach discussed in [94]. 

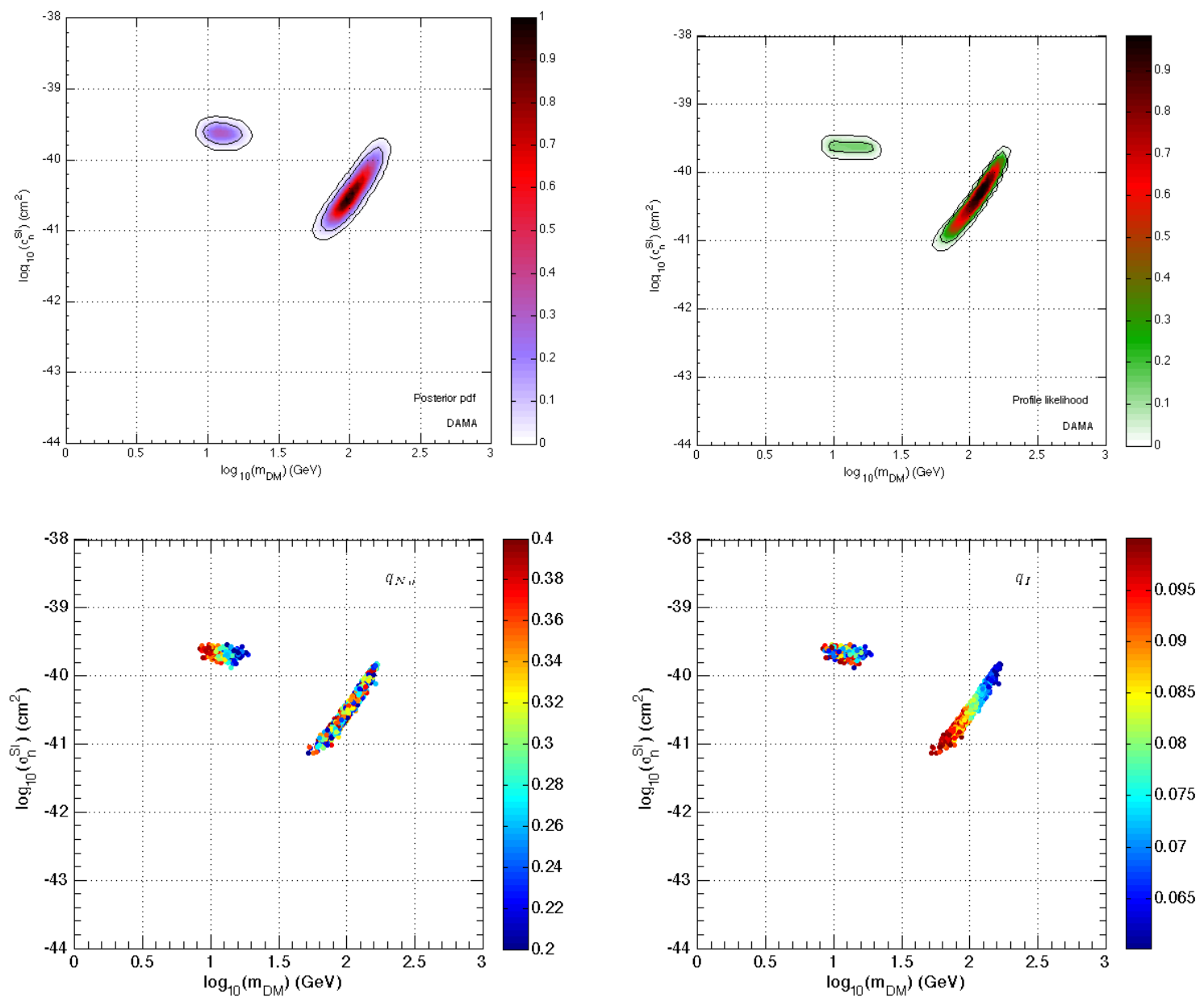

Figure 1. Inference for DAMA assuming the SMH. Top left: 2D marginal posterior pdf in the $\left\{m_{\mathrm{DM}}, \sigma_{n}^{\mathrm{SI}}\right\}$-plane. The black solid lines enclose the $90 \%$ and the $99 \%$ credible regions. Top right: Profile likelihood in the $\left\{m_{\mathrm{DM}}, \sigma_{n}^{\mathrm{SI}}\right\}$-plane. The black solid contours correspond to $\Delta \chi_{\text {eff }}^{2}=4.6,9.2$. Bottom left: 3D marginal posterior pdf for $\left\{m_{\mathrm{DM}}, \sigma_{n}^{\mathrm{SI}}, q_{\mathrm{Na}}\right\}$, where the $q_{\mathrm{Na}}$ direction is represented by the colour code. Bottom right: Same as bottom left, but for $\left\{m_{\mathrm{DM}}, \sigma_{n}^{\mathrm{SI}}, q_{\mathrm{I}}\right\}$.

$\mathcal{L}_{\text {prof }}\left(m_{\mathrm{DM}}, \sigma_{n}^{\mathrm{SI}}\right)$ coincide to an excellent degree, suggesting that the nuisance directions contribute no strong volume effects. For the profile likelihood, we also plot two $\Delta \chi_{\text {eff }}^{2}$ contours, defined via

$$
\Delta \chi_{\text {eff }}^{2}\left(m_{\mathrm{DM}}, \sigma_{n}^{\mathrm{SI}}\right) \equiv-2 \ln \mathcal{L}_{\text {prof }}\left(m_{\mathrm{DM}}, \sigma_{n}^{\mathrm{SI}}\right),
$$

where the choice of $\Delta \chi_{\text {eff }}^{2}=4.6,9.2$ coincides with the classical $90 \%$ and $99 \%$ confidence intervals for two degrees of freedom (assuming Wilks' theorem holds). Again, we find remarkable agreement between these contours and the $90 \%$ and $99 \%$ credible regions inferred from the volume of the 2D marginal posterior. This agreement indicates that when the data are sufficiently informative so that the likelihood function overcomes the dependence on the priors, Bayesian and classical statistical methods yield very similar inference results. 

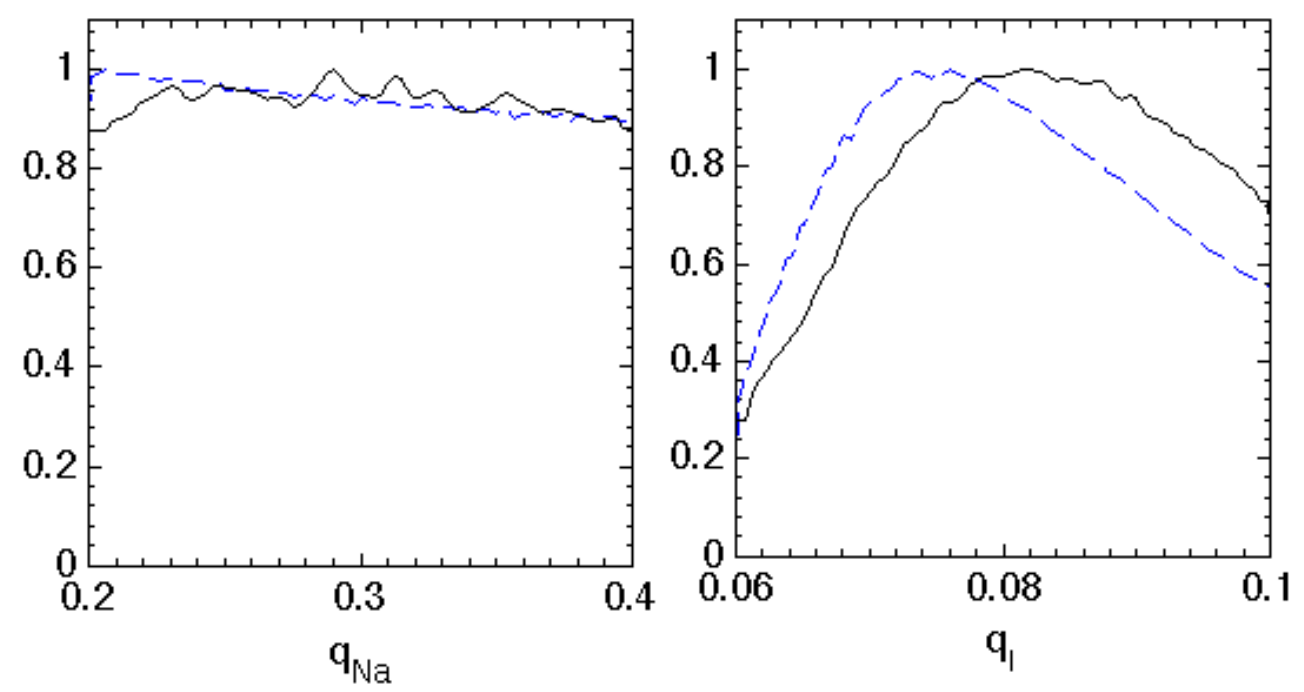

Figure 2. Nuisance parameters for DAMA assuming the SMH. Left: 1D marginal posterior pdf (black solid line) and profile likelihood (blue dashed line) for the quenching factor $q_{\mathrm{Na}}$. Right: Same as left panel, but for $q_{\mathrm{I}}$.

The bottom panel of figure 1 illustrates the correlation between $\left\{m_{\mathrm{DM}}, \sigma_{n}^{\mathrm{SI}}\right\}$ and the quenching factors $q_{\mathrm{Na}}$ and $q_{\mathrm{I}}$. As expected, the high mass $\left(m_{\mathrm{DM}} \sim \mathcal{O}(100) \mathrm{GeV}\right)$ island is insensitive to $q_{\mathrm{Na}}$, as indicated by the equal representation of $q_{\mathrm{Na}}$ values in the island. Conversely, the low mass $\left(m_{\mathrm{DM}} \sim \mathcal{O}(10) \mathrm{GeV}\right)$ island shows a strong correlation between $q_{\mathrm{Na}}$ and $m_{\mathrm{DM}}$, with higher values of $q_{\mathrm{Na}}$ favouring the lower masses. The quenching factor for iodine shows the opposite trend: the low mass island is insensitive to $q_{\mathrm{I}}$, while the high mass island finds combinations of low $m_{\mathrm{DM}}$ and $\sigma_{n}^{\mathrm{SI}}$ values favoured by large values of $q_{\mathrm{I}}$. Ultimately, however, the DAMA 36-bin data do not constrain either $q_{\mathrm{Na}}$ or $q_{\mathrm{I}}$, as is evidenced by the fact that all values of $q_{\mathrm{Na}}$ and $q_{\mathrm{I}}$ allowed by their respective priors are represented in figure 1 . The same conclusions can be drawn also from figure 2, which shows an essentially flat 1D marginal posterior pdf (black solid line) and profile likelihood (dashed blue line) for $q_{\mathrm{Na}}$, while for $q_{\mathrm{I}}$ one might claim a small preference for $q_{\mathrm{I}}=0.07 \rightarrow 0.08$ although it is statistically insignificant.

CoGeNT Figure 3 shows the preferred $\left\{m_{\mathrm{DM}}, \sigma_{n}^{\mathrm{SI}}\right\}$, both in terms of the $2 \mathrm{D}$ marginal posterior pdf and the profile likelihood. As in the case of DAMA, the nuisance directions do not contribute strong volume effects, so that both the $90 \%$ and $99 \%$ credible regions inferred from the marginal posterior coincide well with the $\Delta \chi_{\text {eff }}^{2}=4.6,9.2$ contours on the profile likelihood surface, and single out a peak at $m_{\mathrm{DM}} \sim 8 \mathrm{GeV}$ and $\sigma_{n}^{\mathrm{SI}} \sim 10^{-40} \mathrm{~cm}^{2}$ as the favoured region. The preferred values for the nuisance parameters are reported in table 4. Our analysis is compatible with all previous analyses of the CoGeNT data, and also with the newest data release [95], which claims detection of an annual modulation and where the total rate excess leads to a slightly smaller region in the $\left\{m_{\mathrm{DM}}, \sigma_{n}^{\mathrm{SI}}\right\}$-plane. 

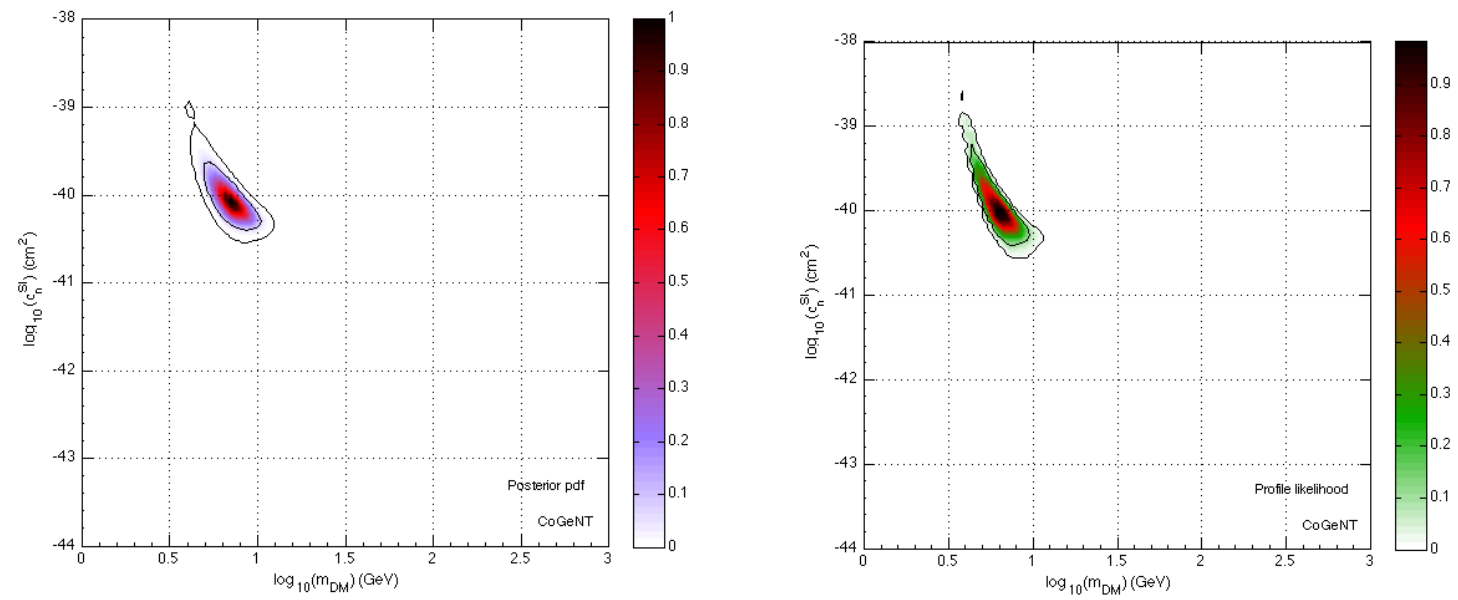

Figure 3. Inference for CoGeNT assuming the SMH. Left: 2D marginal posterior pdf in the $\left\{m_{\mathrm{DM}}, \sigma_{n}^{\mathrm{SI}}\right\}$-plane. The black solid lines enclose the $90 \%$ and the $99 \%$ credible regions. Right: Profile likelihood in the $\left\{m_{\mathrm{DM}}, \sigma_{n}^{\mathrm{SI}}\right\}$-plane. The black solid contours correspond to $\Delta \chi_{\text {eff }}^{2}=4.6,9.2$.

Table 4. 1D marginal posterior pdf modes and 90\% credible intervals for the CoGeNT nuisance parameters.

\begin{tabular}{ll}
\hline Parameter & Preferred value \\
\hline $\mathcal{E}_{0}$ & $6.1_{-5.0}^{+13.1} \mathrm{keV}$ \\
$C$ & $4.0_{-2.3}^{+4.8} \mathrm{cpd} / \mathrm{kg} / \mathrm{keV}$ \\
$G_{n}$ & $2.1 \pm 0.5 \mathrm{cpd} / \mathrm{kg} / \mathrm{keV}$ \\
\hline
\end{tabular}

Xenon100 Our inference results for Xenon100 are shown in figure 4. Firstly, we note that both the 2D marginal posterior pdf and the profile likelihood form a plateau as $m_{\mathrm{DM}}$ and $\sigma_{n}^{\mathrm{SI}}$ approach their respective lower boundaries. ${ }^{+}$In this case, credible regions constructed from the volume of the marginal posterior in $\left\{m_{\mathrm{DM}}, \sigma_{n}^{\mathrm{SI}}\right\}$-space can be strongly dependent on our choice of the $m_{\mathrm{DM}}$ and $\sigma_{n}^{\mathrm{SI}}$ prior boundaries. This is illustrated in the left panel of figure 4 and in figure 5. In both figures the $90 \%$ credible region is demarcated by the black solid line, except that in figure 5 we have chosen a set of prior boundaries for $m_{\mathrm{DM}}$ and $\sigma_{n}^{\mathrm{SI}}\left(0.5 \leq \log \left(m_{\mathrm{DM}} / \mathrm{GeV}\right) \leq 2\right.$ and $\left.-45 \leq \log \left(\sigma_{n}^{\mathrm{SI}} / \mathrm{cm}^{2}\right) \leq-39\right)$ differing from the default choices of table 1 . The discrepancy between the encompassed parameter space is clear. As an example, while the point $\left\{\log \left(m_{\mathrm{DM}} / \mathrm{GeV}\right)=0.8, \log \left(\sigma_{n}^{\mathrm{SI}} / \mathrm{cm}^{2}\right)=-40\right\}$ sits outside the $90 \%$ credible region in figure 4 , it sits comfortably within in figure 5 .

+ Strictly speaking, the profile likelihood shown in figure 4 for Xenon100 is a quasi-profile likelihood, computed after the full likelihood function (4.21) has been analytically marginalised over the background uncertainties. 

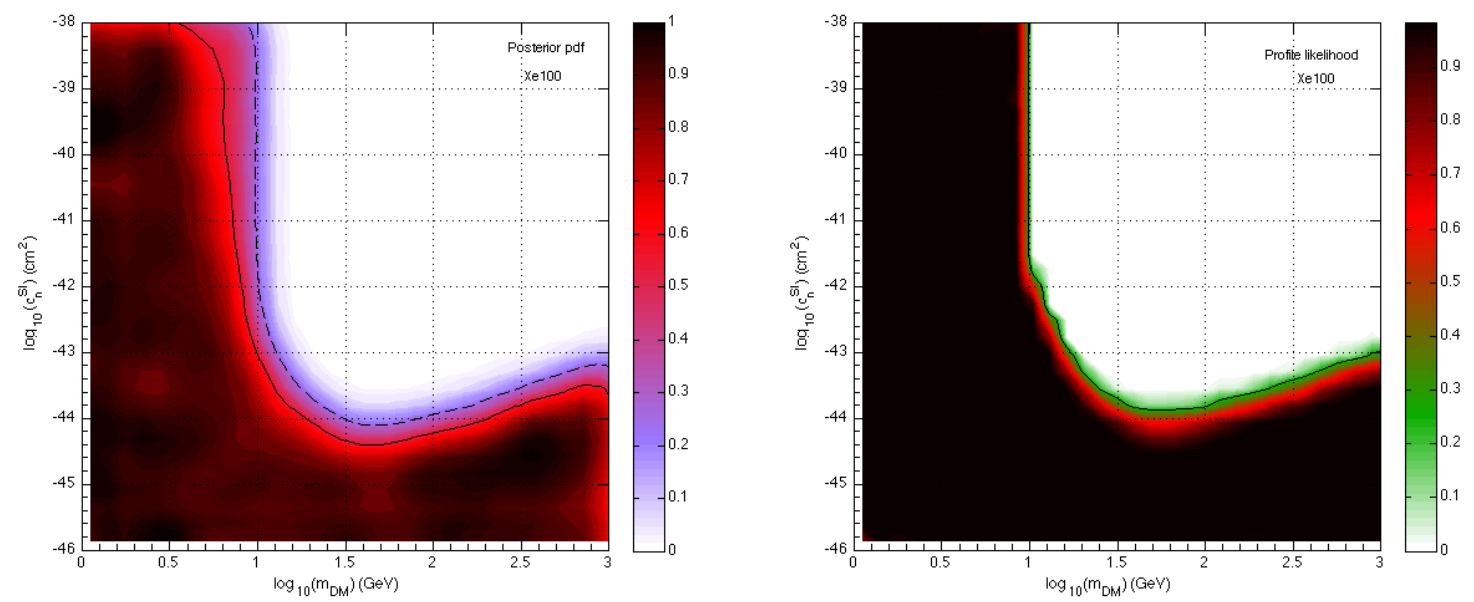

Figure 4. Inference for Xenon100 assuming the SMH. Left: 2D marginal posterior pdf in the $\left\{m_{\mathrm{DM}}, \sigma_{n}^{\mathrm{SI}}\right\}$-plane. The black solid line indicates the $90 \%$ bound inferred from the volume of the marginal posterior, while the black dashed line denotes the invariant $90_{S} \%$ contour. Right: Profile likelihood in the $\left\{m_{\mathrm{DM}}, \sigma_{n}^{\mathrm{SI}}\right\}$-plane. The black dashed line corresponds to $\Delta \chi_{\text {eff }}^{2}=2.7$.

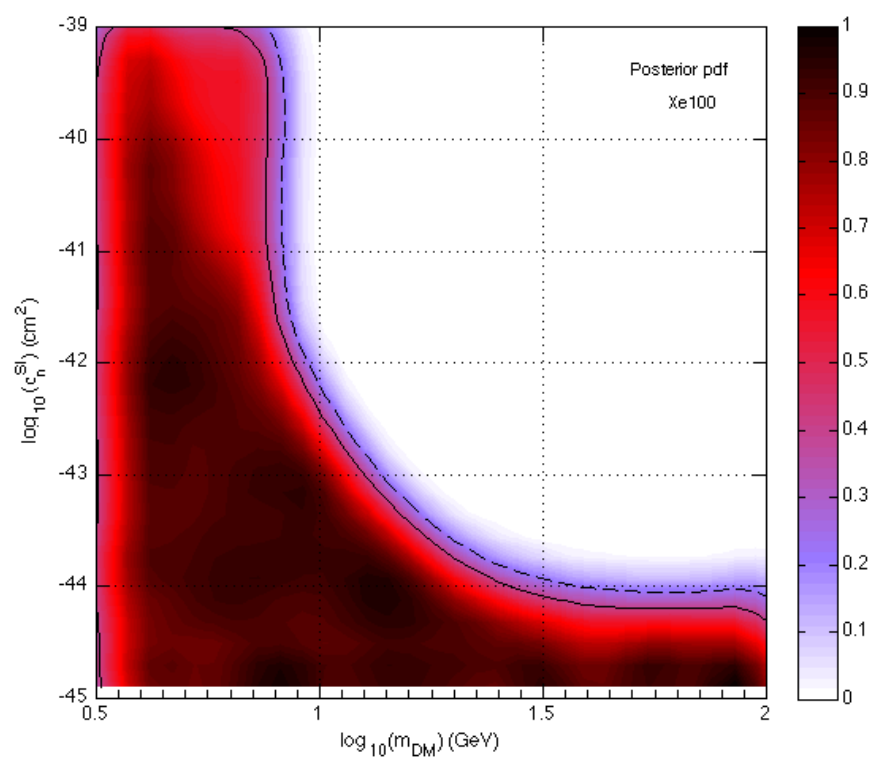

Figure 5. 2D marginal posterior pdf in the $\left\{m_{\mathrm{DM}}, \sigma_{n}^{\mathrm{SI}}\right\}$-plane for Xenon100 assuming the SMH and an alternative set of prior boundaries for $m_{\mathrm{DM}}$ and $\sigma_{n}^{\mathrm{SI}}$. The black solid line corresponds to the $90 \%$ bound inferred from the volume of the marginal posterior, while the black dashed line represents the invariant $90_{S} \%$ contour.

On the other hand, the $90_{S} \%$ bound (black dashed line in left panel of figure 4 and in figure 5) is clearly independent of the boundary conditions as discussed in section 5.2, and the parameter region enclosed compares well with the $\Delta \chi_{\text {eff }}^{2} \leq 2.7$ (or $S \leq 5.2$ ) region in the profile likelihood (right panel of figure 4). We will therefore use the $90_{S} \%$ 


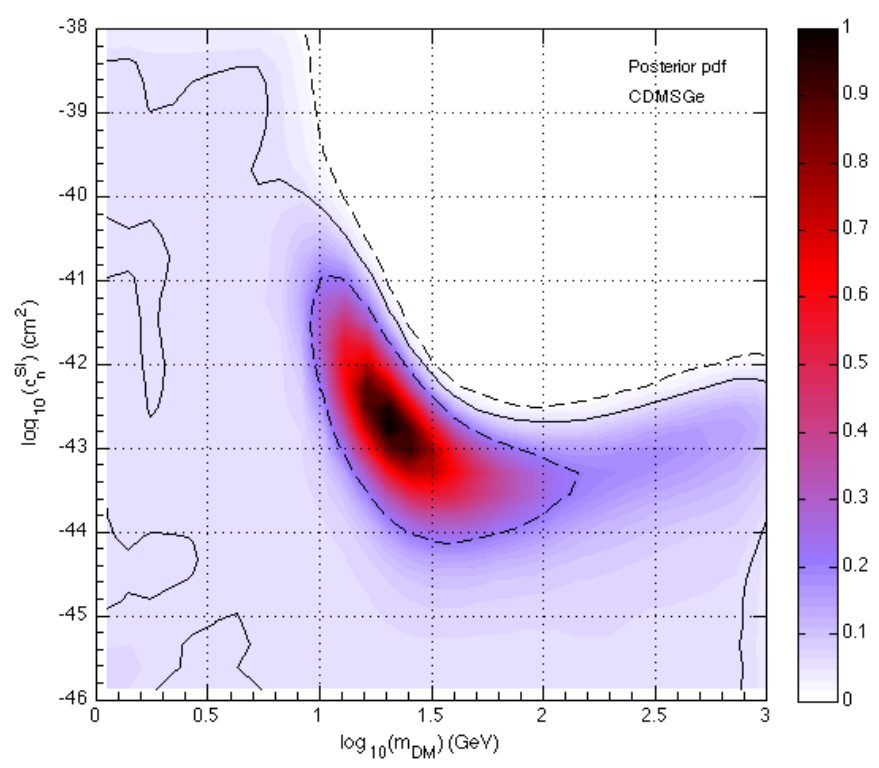

Figure 6. Posterior pdf for CDMSGe assuming the SMH in the $\left\{m_{\mathrm{DM}}, \sigma_{n}^{\mathrm{SI}}\right\}$ plane. The black solid line indicates the $90 \%$ bound inferred from the volume of the posterior, while the black dashed lines denote the invariant $90_{S} \%$ and $99_{S} \%$ contours (corresponding to $\Delta \chi_{\text {eff }}^{2}=3,7.4$ ).

bound in the following discussion.

Our exclusion limit on $\sigma_{n}^{\mathrm{SI}}$ at high WIMP masses $\left(m_{\mathrm{DM}} \gtrsim 30 \mathrm{GeV}\right)$ agrees very well with that provided by the Xenon100 collaboration [19]. However, at low WIMP masses, our bound on $m_{\mathrm{DM}}$ is much less constraining compared with all previous analyses $[7,8$, 19, 25, 96]. This is clearly a consequence of the uncertainties in the scintillation efficiency $\mathrm{L}_{\mathrm{eff}}(E)$ in the low recoil energy $(1<E / \mathrm{keVnr}<3)$ region, which we have accounted for in this work using the nuisance parameter $m$. ${ }^{*}$ The preferred value for this parameter is $m=0.07 \pm 0.04$ (90\% C.I.), which corresponds to a marginal preference for a gentler slope for $\mathrm{L}_{\mathrm{eff}}(\mathrm{E})$ at $1<E / \mathrm{keVnr}<3$ with respect to the Xenon100 collaboration's best-fit.

CDMSGe The posterior pdf as a function of $m_{\mathrm{DM}}$ and $\sigma_{n}^{\mathrm{SI}}$ is shown in figure 6 . Since there are no nuisance parameters - besides the background uncertainty which we have already marginalised analytically in order to obtain the effective likelihood (4.7), the posterior pdf in figure $i s$ the full posterior pdf of the problem. It also coincides with the effective likelihood (4.7) because of our choice of uniform priors. A peak can be seen at a DM mass of $23 \mathrm{GeV}$ and a cross-section of $9 \times 10^{-44} \mathrm{~cm}^{2}$. While this is a tantalising hint, a detection cannot be called because the probability density is still significant at

* We note that the exclusion limits reported by the Xenon collaboration in [19] and [66] are in fact 1D limits on $\sigma_{m}^{\text {SI }}$ for fixed values of $m_{\mathrm{DM}}$. These limits are naturally different from our 2D limits for $\left\{m_{\mathrm{DM}}, \sigma_{n}^{\mathrm{SI}}\right\}$, which come from considering the joint probability distribution of $m_{\mathrm{DM}}$ and $\sigma_{n}^{\mathrm{SI}}$. 


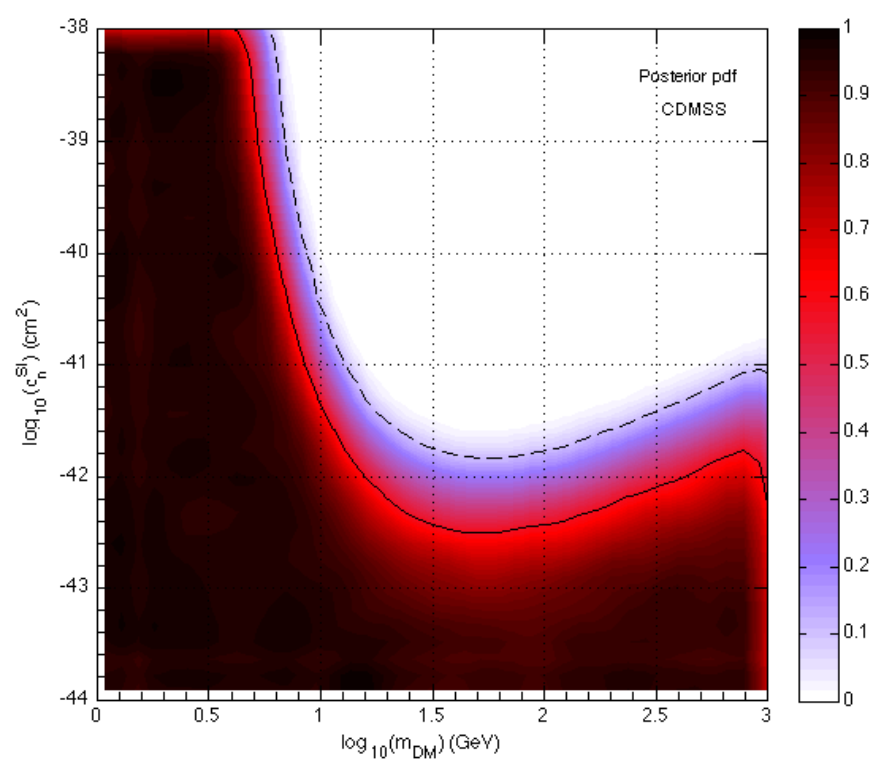

Figure 7. Posterior pdf for CDMSSi assuming the SMH in the $\left\{m_{\mathrm{DM}}, \sigma_{n}^{\mathrm{SI}}\right\}$-plane. The black solid line indicates the $90 \%$ bound inferred from the volume of the posterior, while the black dashed line denotes the invariant $90_{S} \%$ contour (corresponding to $\left.\Delta \chi_{\text {eff }}^{2}=4.2\right)$.

much of the prior boundaries $(\mathcal{P} \sim 0.1)$.

It then remains for us to set an exclusion limit in the $\left\{m_{\mathrm{DM}}, \sigma_{n}^{\mathrm{SI}}\right\}$-plane. The $90 \%$ contour inferred from the volume of posterior (black solid line) forms a semi-closed region subject strongly to our choice of prior boundaries. The invariant $90{ }_{S} \%$ or $\Delta \chi_{\text {eff }}^{2} \leq 3.0$ region (black dashed line), however, is a closed island in the $\left\{m_{\mathrm{DM}}, \sigma_{n}^{\mathrm{SI}}\right\}$-plane, while the $99_{S} \%\left(\Delta \chi_{\text {eff }}^{2}=7.4\right)$ contour indicates an exclusion limit.

Compared with the analysis in figure 3 of [24], our posterior pdf/likelihood appears to be more strongly peaked relative to the plateau, leading to a closed $90_{S} \%$ region while [24] finds an open one. At the same time, our peak region appears to be much broader than that of [24], so that their $90 \%$ contour runs right into our peak region where the posterior pdf/likelihood is still high $(>0.5)$. Fixing the number of background events to the mean value, i.e., setting $\sigma_{B}=0$ in the effective likelihood (4.7), does not ameliorate the discrepancy. Since reference [24] does not specify the likelihood function used in their analysis, we have no more handle to trace the origin of the disagreement.

CDMSSi The analysis of the CDMSSi data is summarised in figure 7. Similar to CDMSGe, the posterior pdf presented in the figure is the full posterior pdf of the problem (barring analytic marginalisation over the background) and coincides with the effective likelihood (4.4). As in the case of Xenon100, the CDMSSi data are not sufficiently constraining to isolate a preferred region, so that the $90 \%$ credible region inferred from the posterior volume (black solid line) depends on our choice of prior boundaries. On 


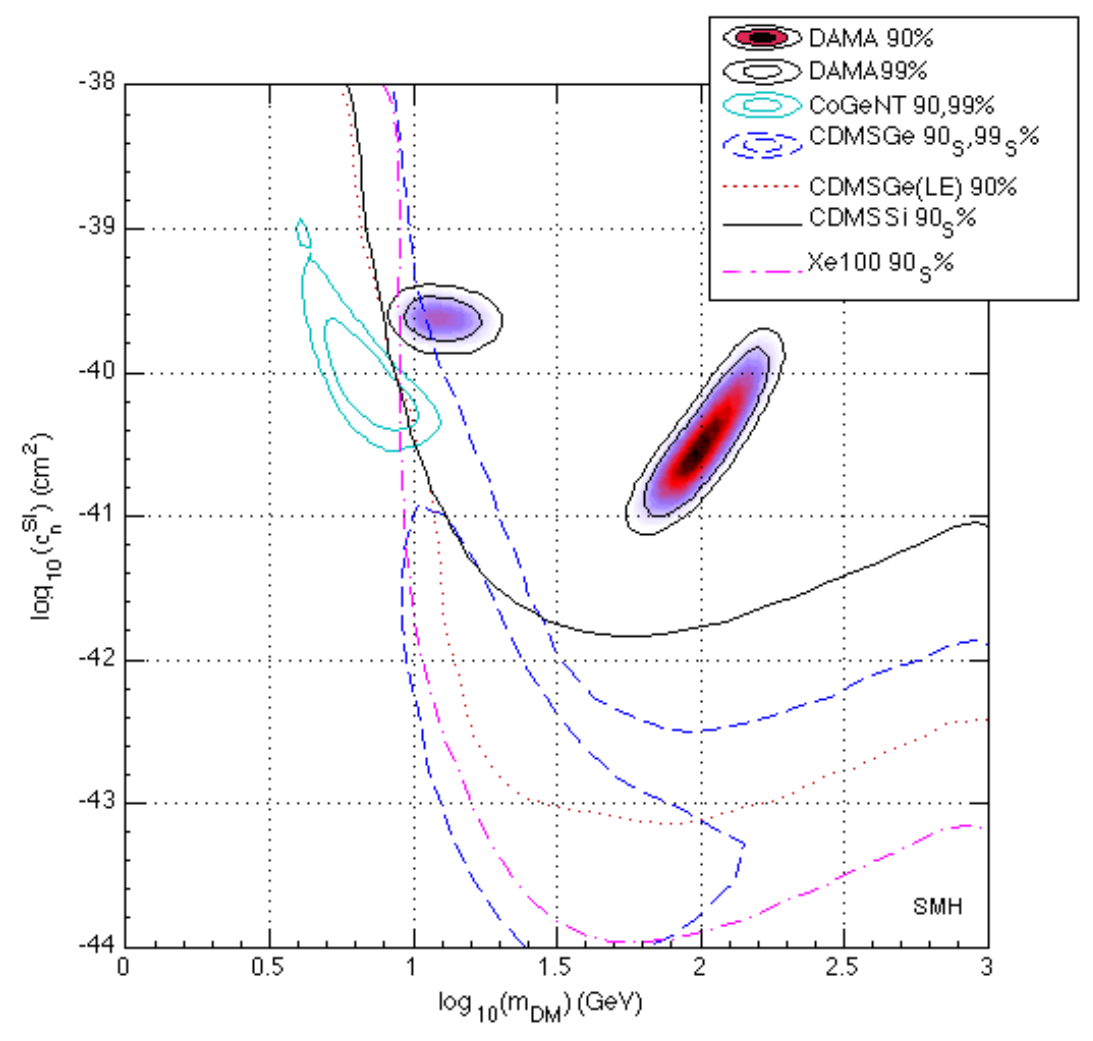

Figure 8. 2D credible regions for the individual experimental bounds and regions assuming the SMH, combined in a single plot. For DAMA (shaded) and CoGeNT (cyan) we show the $90 \%$ and $99 \%$ contours. The black solid line represents the $90_{S} \%$ bound for CDMSSi, and the pink dot-dash curve for Xenon100. For CDMSGe we show both the $90_{S} \%$ and $99_{S} \%$ contours in blue dashed lines, while the red dotted line is the $90 \%$ contour for CDMSGe(LE) corresponding to $\Delta \chi_{\text {eff }}^{2}=4.6$.

the other hand, the $90_{S} \%$ region (black dashed line, corresponding to $\Delta \chi_{\text {eff }}^{2} \leq 4.2$ or $S \leq 3.3)$ is independent of the $m_{\mathrm{DM}}$ and $\sigma_{n}^{\mathrm{SI}}$ prior boundaries, and agrees well with the exclusion limit constructed by the CDMS collaboration [17].

SMH state of the art We summarise our results for fixed astrophysics in figure 8, in which we show all experimental constraints in one plot. For DAMA and CoGeNT we indicate the $90 \%$ and $99 \%$ credible regions, while for the exclusion limits of the other three experiments we show the invariant $90_{S} \%$ contours (also $99_{S} \%$ for CDMSGe).

We find that the parameter region favoured by DAMA is incompatible with the $90_{S} \%$ credible regions of Xenon 100 and CDMSSi, and partially allowed by the $99_{S} \%$ region of CDMSGe. In contrast, the CoGeNT preferred region is only marginally incompatible with these exclusion limits. Of particular interest is the compatibility between CoGeNT and Xenon100. While the Xenon100 collaboration claims that their exclusion limit has ruled out the CoGeNT preferred region [19], we find that when uncertainties in the scintillation efficiency $\mathrm{L}_{\mathrm{eff}}(E)$ at low recoil energies are accounted for, the CoGeNT and the Xenon100 data can find some common ground. 
Between CoGeNT and DAMA we find that their $99 \%$ credible regions do not overlap, despite marginalisation over the quenching factors $q_{\mathrm{Na}}$ and $q_{\mathrm{I}}$ for DAMA. This is a consequence of our choice of prior boundaries for $q_{\mathrm{Na}}(0.2 \rightarrow 0.4)$, especially in view of $[6,7]$, where it has been suggested that in order to make DAMA and CoGeNT compatible large quenching factors for sodium (e.g., $q_{\mathrm{Na}}=0.6$ ) and for germanium should be considered. Allowing up to $10 \%$ of channelling for DAMA could also improve the agreement between the two experiments, by shifting the DAMA low mass region downwards in the $\left\{m_{\mathrm{DM}}, \sigma_{n}^{\mathrm{SI}}\right\}$-plane [69].

Lastly, we also show the exclusion bound derived from CDMSGe(LE) (red dotted line in figure 8). Since the likelihood function (4.11) for this case is a multivariate gaussian, we can infer an invariant $90 \%$ exclusion bound similar to the $90_{S} \%$ bound by demanding that $\Delta \chi_{\text {eff }}^{2}<4$.6. At low masses this $90 \%$ bound turns out to be very close to the CDMSSi exclusion limit, so that the DAMA preferred region falls outside the credible region, while the CoGeNT region falls mostly within. The main difference between this low energy analysis and the standard CDMSGe is that the former does not find any closed region at small WIMP masses. Compared with other experiments, for masses larger than $10 \mathrm{GeV}$ the Xenon100 bound is more constraining. We therefore do not consider the CDMSGe(LE) exclusion limit any further.

\subsection{Variable WIMP velocity distribution and astrophysics}

Figure 9 shows the effects of astrophysical uncertainties on the inferred $\left\{m_{\mathrm{DM}}, \sigma_{n}^{\mathrm{SI}}\right\}$ parameter space, for each of the four DM density profiles considered (see section 3.1). The corresponding preferred values for the local dark matter density, the circular and the escape velocities are reported in table 5 .

Firstly, we note that all four DM profiles give very similar inference results on the

$\left\{m_{\mathrm{DM}}, \sigma_{n}^{\mathrm{SI}}\right\}$-plane. This means that the exact shape of the DM halo density profile - at least within the class of spherically symmetric, smooth profiles - does not yet play a role in direct DM searches. This conclusion is further supported by the inferred local DM density, circular and escape velocities presented in table 5. The preferred values for these quantities differ from profile to profile, with the Cored isothermal halo in particular favouring the very high end of the observationally allowed escape velocities (see table 2). However, once the DM halo profile has been fixed, we see that the preferred values for $v_{0}$, $v_{\text {esc }}$ and $\rho_{\odot}$ and their associated uncertainties are virtually independent of the additional constraints from the DM experiments. In other words, direct DM searches are not at the moment contributing towards constraining the astrophysics of the problem.

Secondly, we note that allowing for uncertainties in the astrophysics significantly expands the closed regions of DAMA, CDMSGe and CoGeNT, while the exclusion limits tend to shift a little to the right. For all four profiles, the preferred regions of DAMA and CoGeNT now appear to marginally overlap: for the NFW, Einasto and Burkert profiles we see an overlap between the 90\% credible region of DAMA with the $99 \%$ region of CoGeNT and vice versa, while for the Cored isothermal the agreement is a 

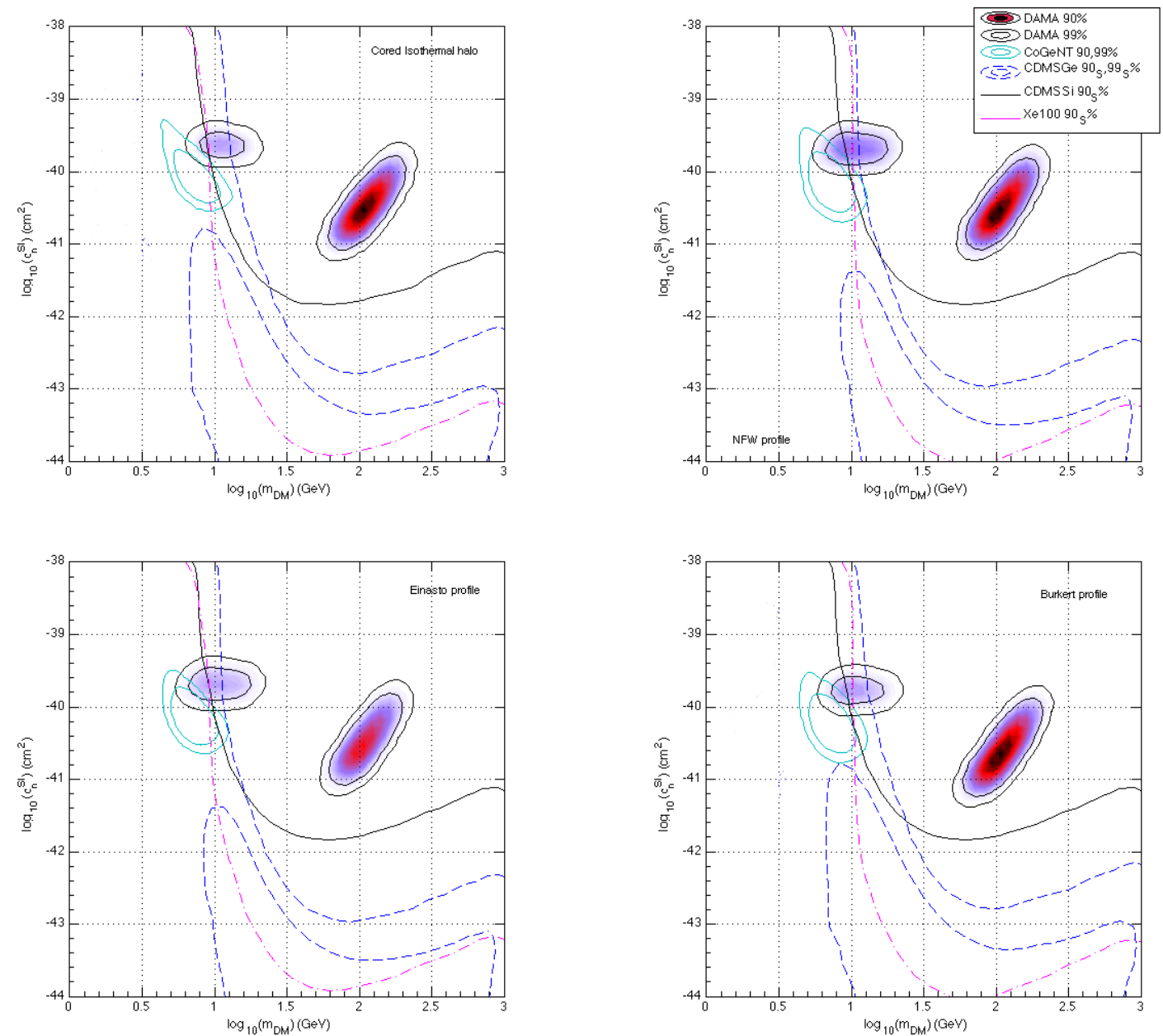

Figure 9. Same as figure 8, but with variable astrophysics, assuming the Cored isothermal (top left), NFW (top right), Einasto (bottom left), and Burkert (bottom right) profiles.
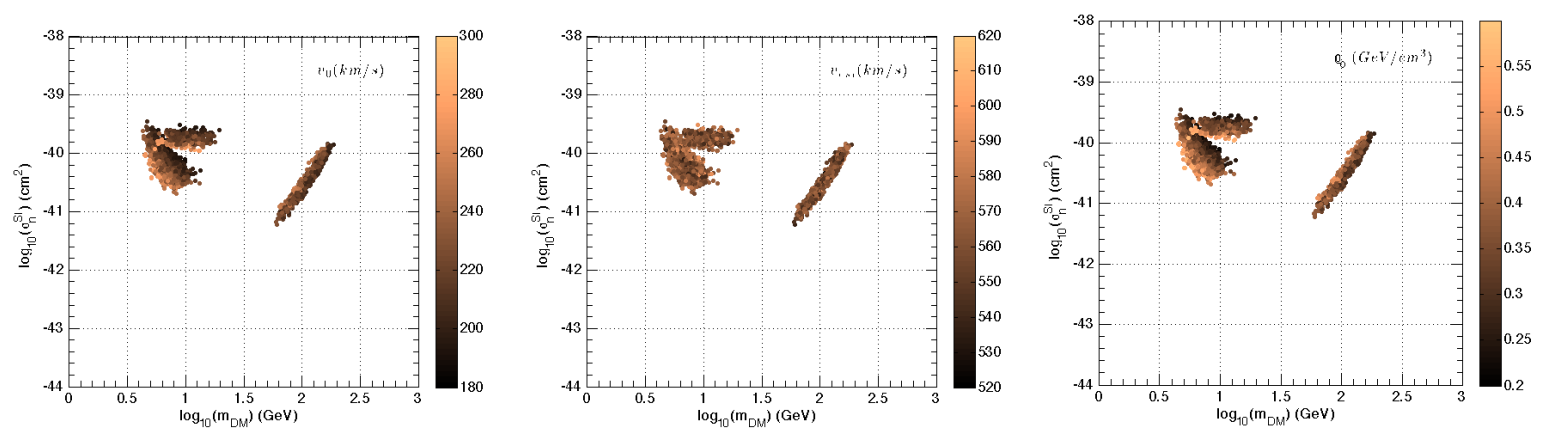

Figure 10. 3D marginal posterior pdf for DAMA and CoGeNT for $\left\{m_{\mathrm{DM}}, \sigma_{n}^{\mathrm{SI}}\right\}$ and the circular velocity $v_{0}$ (left), the escape velocity $v_{\text {esc }}$ (centre), and the local DM density $\rho_{\odot}$ (right), assuming the NFW profile. The third parameter direction is represented by the colour code. 
Bayes and present DM direct detection

Table 5. 1D posterior pdf modes and $90 \%$ credible intervals for the circular velocity $v_{0}$, escape velocity $v_{\text {esc }}$, and the local DM density $\rho_{\odot}$ for DM density profiles considered in this work.

\begin{tabular}{l|lll}
\hline & $v_{0}\left(\mathrm{~km} \mathrm{~s}^{-1}\right)$ & $v_{\text {esc }}\left(\mathrm{km} \mathrm{s}^{-1}\right)$ & $\rho_{\odot}\left(\mathrm{GeV} \mathrm{cm} \mathrm{cm}^{-3}\right)$ \\
\hline Cored Isothermal & & & \\
DAMA & $210_{-16}^{+26}$ & $628_{-17}^{+22}$ & $0.31_{-0.03}^{+0.05}$ \\
CoGeNT & $209_{-21}^{+14}$ & $628 \pm 18$ & $0.31 \pm 0.04$ \\
CDMSGe & $208_{-16}^{+22}$ & $628_{-21}^{+23}$ & $0.31 \pm 0.05$ \\
CDMSSi & $210_{-16}^{+29}$ & $628 \pm 21$ & $0.31_{-0.04}^{+0.05}$ \\
Xenon100 & $211_{-19}^{+26}$ & $629 \pm 21$ & $0.31 \pm 0.04$ \\
\hline NFW & & & \\
DAMA & $220_{-20}^{+40}$ & $558_{-16}^{+19}$ & $0.37_{-0.09}^{+0.15}$ \\
CoGeNT & $219_{-18}^{+38}$ & $559 \pm 17$ & $0.37_{-0.08}^{+0.20}$ \\
CDMSGe & $218_{-18}^{+41}$ & $559 \pm 18$ & $0.37_{-0.08}^{+0.16}$ \\
CDMSSi & $218_{-19}^{+44}$ & $560_{-18}^{+19}$ & $0.36_{-0.09}^{+0.18}$ \\
Xenon100 & $219_{-20}^{+43}$ & $559 \pm 18$ & $0.37_{-0.08}^{+0.16}$ \\
\hline Einasto & & & \\
DAMA & & & \\
CoGeNT & $221_{-19}^{+39}$ & $560_{-18}^{+13}$ & $0.36_{-0.08}^{+0.14}$ \\
CDMSGe & $222_{-19}^{+42}$ & $562_{-21}^{+11}$ & $0.36_{-0.08}^{+0.15}$ \\
CDMSSi & $221_{-19}^{+44}$ & $561_{-22}^{+11}$ & $0.36_{-0.08}^{+0.15}$ \\
Xenon100 & $221_{-19}^{+44}$ & $561_{-22}^{+11}$ & $0.36_{-0.08}^{+0.15}$ \\
\hline Burkert & $221_{-19}^{+44}$ & $562_{-22}^{+11}$ & $0.36_{-0.08}^{+0.15}$ \\
DAMA & \multicolumn{3}{|}{} \\
CoGeNT & $214_{-21}^{+36}$ & $548_{-16}^{+29}$ & $0.44_{-0.12}^{+0.16}$ \\
CDMSGe & $216_{-22}^{+35}$ & $550 \pm 20$ & $0.44_{-0.12}^{+0.16}$ \\
CDMSSi & $215_{-23}^{+35}$ & $549 \pm 19$ & $0.44_{-0.12}^{+0.18}$ \\
Xenon100 & $215_{-23}^{+35}$ & $550 \pm 22$ & $0.44_{-0.13}^{+0.18}$ \\
\hline & $216_{-23}^{+35}$ & $550 \pm 21$ & $0.44_{-0.13}^{+0.16}$ \\
\hline
\end{tabular}

little worse. One may be tempted to claim some degree of agreement between DAMA and CoGeNT based on this partial overlap. However, before we do so, it is important that we also examine the degree of overlap between the preferred regions in the other parameter directions.

Figure 10 shows the 3D marginal posterior pdf for $\left\{m_{\mathrm{DM}}, \sigma_{n}^{\mathrm{SI}}\right\}$ and a third parameter direction $v_{0}, v_{\text {esc }}$ and $\rho_{\odot}$. Here, we see that while it is not impossible to find a value of $v_{\text {esc }}$ that satisfies both DAMA and CoGeNT simultaneously, there is a clear trend that combinations of larger $\left\{m_{\mathrm{DM}}, \sigma_{n}^{\mathrm{SI}}\right\}$ values tend to prefer higher values of $v_{0}$ (and similarly for $\left.\rho_{\odot}\right)$. This indicates that although DAMA and CoGeNT appear to overlap in the $\left\{m_{\mathrm{DM}}, \sigma_{n}^{\mathrm{SI}}\right\}$-plane, there is in fact very little overlap between them in the $v_{0}$ direction (and naturally also in the $\rho_{\odot}$ direction which enters into the differential recoil rates as a common normalisation factor). 

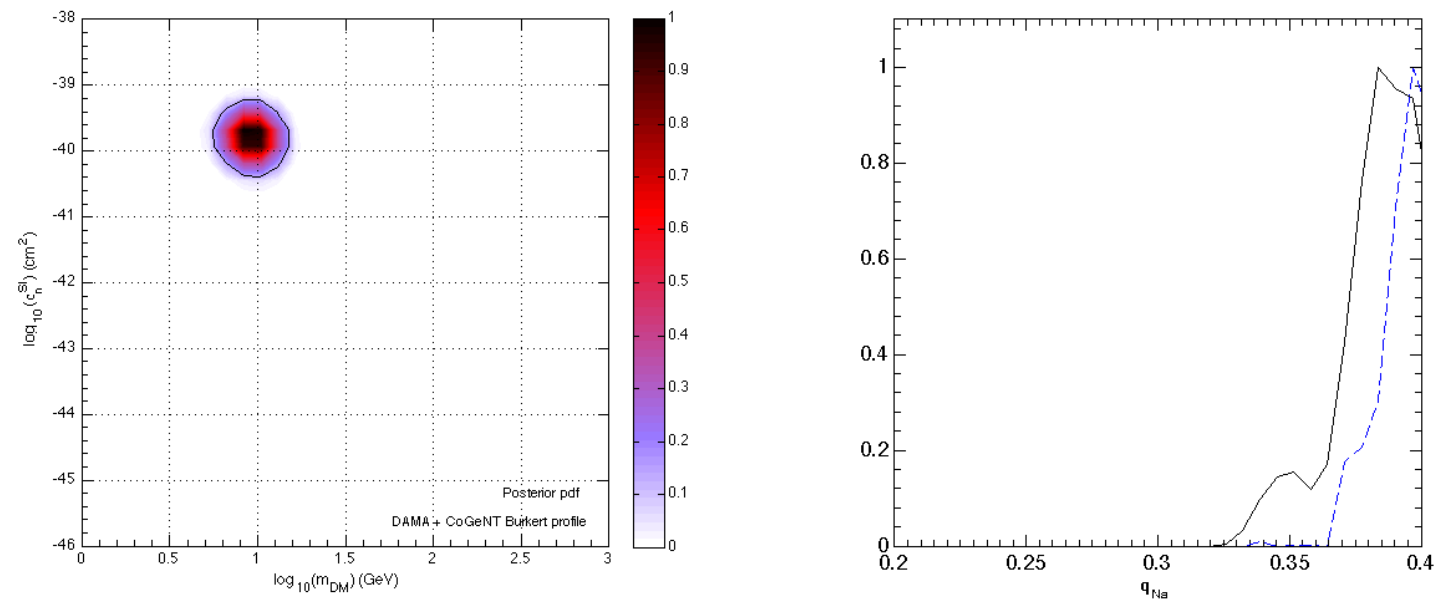

Figure 11. Inference from the combined DAMA and CoGeNT fit assuming the Burkert halo profile. Left: 2D marginal posterior pdf in the $\left\{m_{\mathrm{DM}}, \sigma_{n}^{\mathrm{SI}}\right\}$-plane. The black solid lines correspond to the $90 \%$ and $99 \%$ bound inferred from the volume of the marginal posterior. Right: 1D marginal posterior pdf (black solid line) and profile likelihood (blue dashed line) for the quenching factor $q_{\mathrm{Na}}$.

\subsection{Combined fit?}

Despite apparent difficulties to reconcile the DAMA and the CoGeNT preferred regions within the boundaries of our nuisance and astrophysics models, let us for a moment entertain the possibility of a combined fit. Figure 11 shows the preferred region in the $\left\{m_{\mathrm{DM}}, \sigma_{n}^{\mathrm{SI}}\right\}$-plane from a combined fit of DAMA and CoGeNT assuming the Burkert profile (marginalised over all nuisance and astrophysics parameters as usual). The corresponding 1D marginal credible intervals for the nuisance and astrophysics parameter are displayed in table 6 .

The best-fit point of the combined fit corresponds to a mass of $9.2 \mathrm{GeV}$ and a cross-section of $1.26 \times 10^{-40} \mathrm{~cm}^{2}$. However, this fit comes at the expense of a significant shift in the circular velocity: $v_{0}=176_{-1}^{+33} \mathrm{~km} \mathrm{~s}^{-1}$ (90\% C.I.) from the combined fit, versus $v_{0}=214_{-21}^{+36} \mathrm{~km} \mathrm{~s}^{-1}$ from fitting either DAMA or CoGeNT alone. The preferred local DM density $\rho_{\odot}$ and escape velocity $v_{\text {esc }}$ also suffer a downward shift respectively, although not a significant one in either case. For the nuisance parameters, we find that for CoGeNT, the normalisation for the exponentially decaying background $C$ has come down a little, and the decay rate $\mathcal{E}_{0}$ is significantly more constrained. The radiative peaks, on the other hand, are a well-defined feature, and consequently the preferred value for their height $G_{n}$ has not been affected by the combined fit.

Most interestingly, we find that the DAMA sodium quenching factor $q_{\mathrm{Na}}$, which was previously an unconstrained quantity (see figure 2), now shows a preference for high values (right panel of figure 11). In particular, the 1D profile likelihood (blue dashed line) has no local maximum, and hits its highest point right at the prior boundary 
Table 6. 1D marginal posterior pdf modes and $90 \%$ credible intervals for the astrophysical and the nuisance parameters from the combined DAMA and CoGeNT fit assuming the Burkert profile.

\begin{tabular}{ll}
\hline Parameter & Preferred value \\
\hline$v_{0}$ & $176_{-1}^{+33} \mathrm{~km} \mathrm{~s}^{-1}$ \\
$v_{\text {esc }}$ & $533_{-8}^{+27} \mathrm{~km} \mathrm{~s}^{-1}$ \\
$\rho_{\odot}$ & $0.3_{-0.09}^{+0.2} \mathrm{GeV} \mathrm{cm}^{-3}$ \\
$q_{\mathrm{Na}}$ & $0.38_{-0.03}^{+0.02}$ \\
$\mathcal{E}_{0}$ & $5 \pm 1.2 \mathrm{keV}$ \\
$C$ & $2.8_{-1.7}^{+2.8} \mathrm{cpd} / \mathrm{kg} / \mathrm{keV}$ \\
$G_{n}$ & $2.2 \pm 0.4 \mathrm{cpd} / \mathrm{kg} / \mathrm{keV}$ \\
\hline
\end{tabular}

$q_{\mathrm{Na}}=0.4$. This suggests that if we had allowed for a wider prior range for $q_{\mathrm{Na}}$, an even higher value might have been preferred. This result is consistent with previous suggestions that a higher value for $q_{\mathrm{Na}}$ could improve the compatibility of DAMA and CoGeNT (see section 6.4).

To assess the quality of the fit for the best-fit point singled out by the combined run, we look at the spectral shape of the expected signal in both detectors. In figure 12 we show on the left the averaged modulated amplitude of DAMA, and on the right the number of counts per bin versus the recoil energy for CoGeNT. Superimposed here are the predictions for the best-fit point (dashed lines). Clearly, the "best-fit" point is actually a bad fit for both experiments. For the best-fit DM mass, cross-section and nuisance parameters, a better fit in DAMA would be obtained by increasing both $v_{0}$, which would shift the spectral curve to the left, and $\rho_{\odot}$, which would result in a global enhancement of the signal. For CoGeNT the trend is the opposite: a better fit is obtained by decreasing $\rho_{\odot}$ and increasing $v_{0}$, as demonstrated in figure 10 . For both detectors the signal is rather insensitive to the value of $v_{\text {esc }}$.

\subsection{A larger $q_{\mathrm{Na}}$}

As suggested in the previous section, allowing for a larger sodium quenching factor $q_{\mathrm{Na}}$ for DAMA may improve the combined DAMA/CoGeNT fit. We explore this possibility here by raising the upper limit of our prior range on $q_{\mathrm{Na}}$ from 0.4 to 0.6 , and recomputing the preferred regions for combined DAMA/CoGeNT assuming a Burkert profile.

The results are shown in figure 13. On the left panel, the preferred region in the $\left\{m_{\mathrm{DM}}, \sigma_{n}^{\mathrm{SI}}\right\}$-plane is similar to that inferred using our standard prior on $q_{\mathrm{Na}}$ (see figure 11), with the best-fit point now corresponding to a mass of $7.38 \mathrm{GeV}$ and a crosssection of $9.64 \times 10^{-41} \mathrm{~cm}^{2}$. On the right panel, we see that both the $1 \mathrm{D}$ marginal posterior pdf (black solid) and profile likelihood (dashed blue) for $q_{\mathrm{Na}}$ rise sharply 

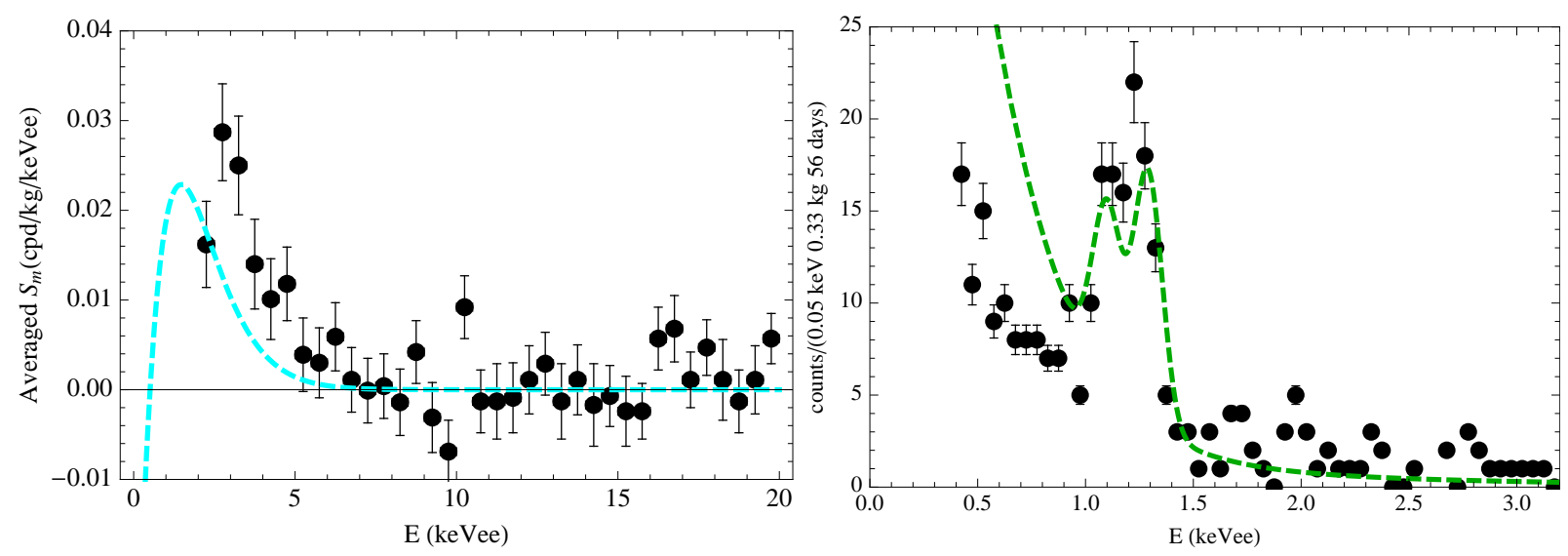

Figure 12. Left: The expected signal for DAMA using the best-fit point of our combined DAMA/CoGeNT fit. Right: The expected signal for CoGeNT using the best-fit point of our combined fit.

between $q_{\mathrm{Na}}=0.5$ and 0.6 , hitting their highest points at the edge of the prior boundary. This confirms the trend that the combined DAMA and CoGeNT data prefer a large $q_{\mathrm{Na}}$.

Interestingly, the preferred values for the astrophysical parameters from the extended combined fit are now more closely in line with those from fits to the individual experiments alone (see table 7). The most significant change can be seen for the circular velocity, which now has the preferred value $v_{0}=201_{-17}^{+35} \mathrm{~km} \mathrm{~s}^{-1}$ (90\% C.I.), in contrast to (a) $v_{0}=176_{-1}^{+33} \mathrm{~km} \mathrm{~s}^{-1}$ from the standard combined fit in section 6.3, and (b) $v_{0}=214_{-21}^{+36} \mathrm{~km} \mathrm{~s}^{-1}$ and $v_{0}=216_{-22}^{+35} \mathrm{~km} \mathrm{~s}^{-1}$ from DAMA and CoGeNT alone respectively.

However, despite this shift in the astrophysical parameters, the extended combined fit offers only a marginal improvement over the standard combined fit. This can be seen in figure 14, where we show the spectral shapes of the expected signals for the individual experiments corresponding to the best-fit point of the extended combined fit. Comparing with figure 12, we see that the fit to CoGeNT now shows better agreement to the data at low energies, while for DAMA the higher value for $q_{\mathrm{Na}}$ now leads to a lower peak in the spectrum, which is further suppressed by the smaller value of $\sigma_{n}^{\mathrm{SI}}$ and the lighter dark matter mass.

\section{Conclusions}

The present status of the direct detection of dark matter is somewhat ambiguous. On the one hand, there have been claims of detection of a low-mass WIMP signal from DAMA and CoGeNT. On the other hand, the Xenon100, CDMS, and CDMS-II experiments have only been able to provide exclusion limits. The interpretation of these experiments in terms of a dark matter signal is complicated by the presence of backgrounds, and the need to model experiment-specific systematic effects, such as the quenching factors for DAMA or the scintillation efficiency for the Xenon detector. In addition, one requires 

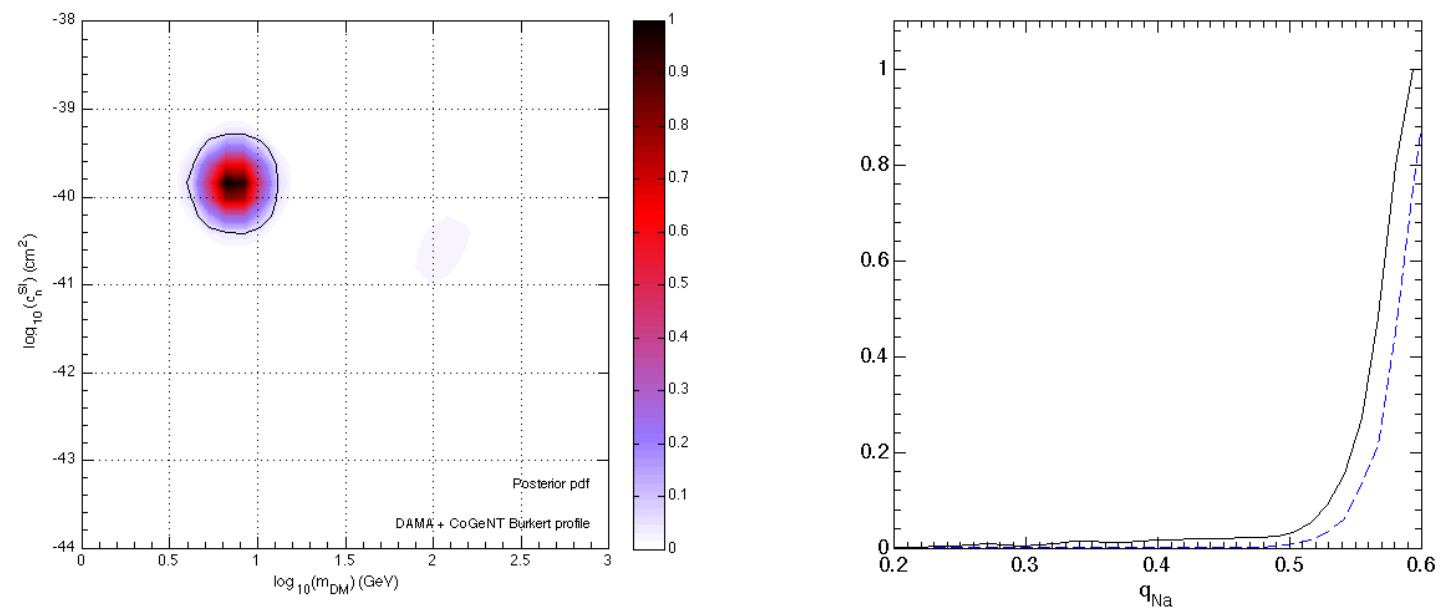

Figure 13. Same as figure 11, but for an extended prior range for the DAMA sodium quenching factor $q_{\mathrm{Na}}$ (up to $q_{\mathrm{Na}}=0.6$ ).

Table 7. Same as table 6, but for an extended prior range for $q_{\mathrm{Na}}$ (up to $q_{\mathrm{Na}}=0.6$ ).

\begin{tabular}{ll}
\hline Parameter & Preferred value \\
\hline$v_{0}$ & $201_{-17}^{+35} \mathrm{~km} \mathrm{~s}^{-1}$ \\
$v_{\text {esc }}$ & $541_{-15}^{+27} \mathrm{~km} \mathrm{~s}^{-1}$ \\
$\rho_{\odot}$ & $0.36_{-0.09}^{+0.2} \mathrm{GeV} \mathrm{cm}^{-3}$ \\
$q_{\mathrm{Na}}$ & $0.59_{-0.04}^{+0.01}$ \\
$\mathcal{E}_{0}$ & $9.4 \pm 1.8 \mathrm{keV}$ \\
$C$ & $3.1_{-1.6}^{+2.9} \mathrm{cpd} / \mathrm{kg} / \mathrm{keV}$ \\
$G_{n}$ & $2.2 \pm 0.4 \mathrm{cpd} / \mathrm{kg} / \mathrm{keV}$ \\
\hline
\end{tabular}

the input of astrophysical quantities, which enter into the theoretical expressions for the total or modulated DM rates. All these effects are to some extent subject to uncertainties, which need to be propagated to the inferred dark matter parameters.

This multi-parameter inference problem can be addressed in a simple and consistent way using Bayesian statistical methods. In the present work, we apply these methods to a selection of current direct dark matter searches to infer the mass and crosssection of WIMP dark matter in the simplest scenario of spin-independent elastic WIMP scattering.

We initially ignore the astrophysical uncertainties and focus on the effects of experimental nuisance parameters and background uncertainties. Our main result is that the Xenon100 exclusion bound is significantly weakened once the uncertainty on the scintillation efficiency is taken into account. As a consequence, we find that the CoGeNT preferred region in the $\left\{m_{\mathrm{DM}}, \sigma_{n}^{\mathrm{SI}}\right\}$-plane is quite compatible with Xenon100, and there is even a marginal consistency (at $90_{S} \%$ credibility) with the DAMA preferred 

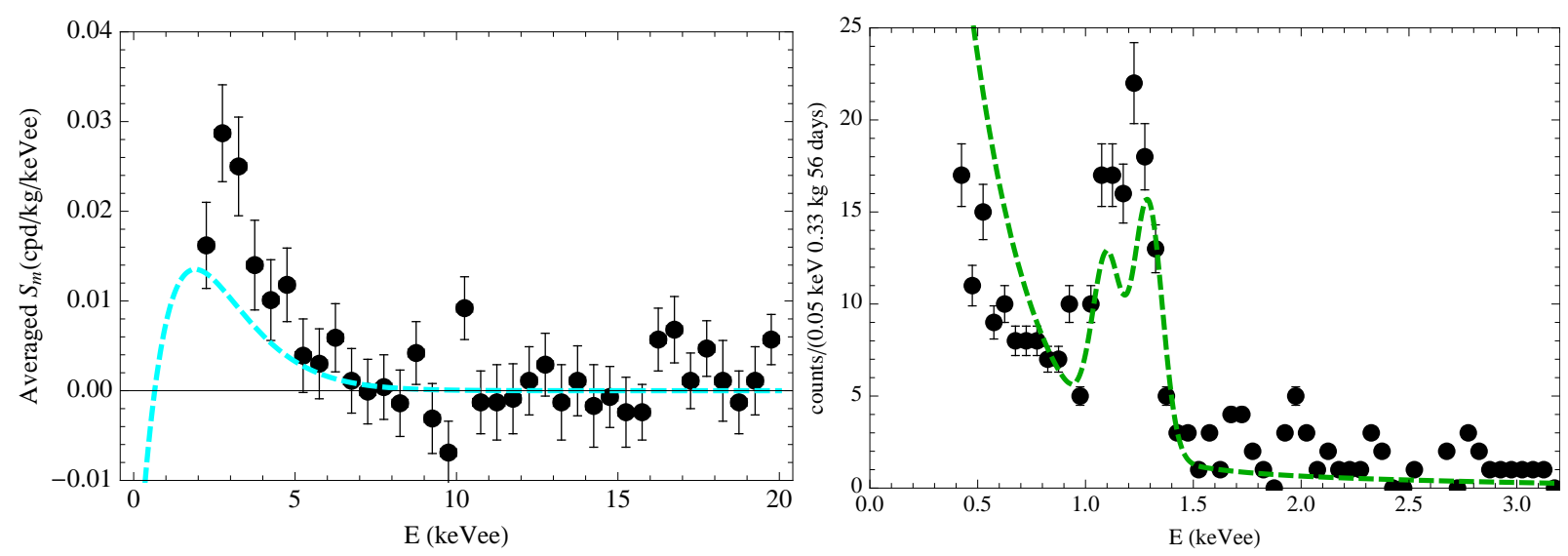

Figure 14. Same as figure 12, but for the best-fit point of the extended combined fit, with $m_{\mathrm{DM}}=7.38 \mathrm{GeV}, \sigma_{n}^{\mathrm{SI}}=9.64 \times 10^{-41} \mathrm{~cm}^{2}$, and other parameter values presented in table 7 .

region. We expect that this conclusion also holds for Xenon10 and the latest CoGeNT data which were obtained after two years of data taking [97]. We also remark that after marginalising over the background, the standard CDMSGe analysis yields a closed $90_{S} \%$-credible contour, although no closed regions remain in the low energy re-analysis CDMSGe(LE).

We then repeat the analysis procedure including astrophysical uncertainties, considering besides the standard model halo three other spherically symmetric dark matter halo models with isotropic velocity distributions, whose density profiles are motivated by $N$-body simulations. We find that the inferred values of the astrophysical parameters are independent of the direct detection experiment data, indicating that their values depend only on the chosen DM density profile. With the exception of the isothermal halo, which prefers significantly higher escape velocities, the different halo parameterisations lead to similar values of the astrophysical parameters. Not unexpectedly, including the astrophysical uncertainties further reduces the constraining power of the data in the $\left\{m_{\mathrm{DM}}, \sigma_{n}^{\mathrm{SI}}\right\}$-plane. At first glance, this seems to improve the compatibility between DAMA and CoGeNT. However, this impression is somewhat misleading, since in some of the marginalised directions $\left(v_{0}\right.$ and $\left.\rho_{\odot}\right)$ the disagreement remains - tweaking astrophysics parameters alone cannot reconcile the two results. If we demand compatibility between these experiments, then the inference process naturally concludes that a high value for the sodium quenching factor for DAMA is preferred.

It will be interesting to apply the analysis framework presented in this paper to more complex models of the dark matter halo, like asymmetric velocity distributions or the presence of streams in the Galactic halo. Additionally, an application to alternative scenarios for the particle physics interactions can be envisaged, such as inelastic DM [4, 95] or more exotic scenarios, as for instance discussed in [4, 24, 98]. 


\section{Acknowledgments}

We thank Roberto Trotta for useful comments on the manuscript. CA acknowledges use of the cosmo computing resources at CP3 of Louvain University. JH gratefully acknowledges support from the Humboldt foundation via a Feodor Lynen-fellowship.

\section{Appendix A. Dark matter density profile in terms of $M_{\mathrm{vir}}$ and $c_{\mathrm{cir}}$}

The two-parameter DM density profiles defined in section 3.1 in terms of $\rho_{s}$ and $r_{s}$ can be expressed in terms of the halo's virial mass $M_{\text {vir }}$ and concentration parameter $c_{\text {vir }}$. Firstly, the parameter $r_{s}$ can be parameterised as

$$
r_{s}\left(M_{\mathrm{vir}}, c_{\mathrm{vir}}\right)=\frac{r_{\mathrm{vir}}\left(M_{\mathrm{vir}}\right)}{c_{\mathrm{vir}}}
$$

where the virial radius $r_{\text {vir }}$ defines a spherical region in which the average DM density is $\delta_{c}=200$ times the critical density $\rho_{\text {crit }}$. The mass enclosed in this region is called the virial mass,

$$
M_{\mathrm{vir}}=4 \pi \int_{0}^{r_{\mathrm{vir}}} \mathrm{d} r r^{2} \rho_{\mathrm{DM}}(r)=\frac{4}{3} \pi r_{\mathrm{vir}}^{3} \delta_{c} \rho_{\text {crit }} .
$$

Using this relation, we can solve for $\rho_{s}$ once a profile has been specified. We give the solutions for the four halo profiles considered in this work:

(i) Cored isothermal:

$$
\rho_{s}\left(c_{\mathrm{vir}}\right)=\frac{\delta_{c} \rho_{\mathrm{crit}}}{3} \frac{c_{\mathrm{vir}}^{3}}{c_{\mathrm{vir}}-\tan ^{-1}\left(c_{\mathrm{vir}}\right)},
$$

(ii) $N F W$ :

$$
\rho_{s}\left(c_{\mathrm{vir}}\right)=\frac{\delta_{c} \rho_{\text {crit }}}{3} \frac{c_{\mathrm{vir}}^{3}}{\ln \left(1+c_{\mathrm{vir}}\right)-c_{\mathrm{vir}} /\left(1+c_{\mathrm{vir}}\right)} .
$$

(iii) Einasto:

$$
\rho_{s}\left(c_{\mathrm{vir}}\right)=\frac{\delta_{c} \rho_{\text {crit }}}{3} \frac{c_{\mathrm{vir}}^{3}\left[2^{-\frac{3}{\alpha}} \exp \left(\frac{2}{\alpha}\right) \alpha^{\frac{3}{\alpha}-1}\right]^{-1}}{\Gamma\left(\frac{3}{\alpha}\right)-\Gamma\left(\frac{3}{\alpha}, \frac{2 c_{\mathrm{vir}}^{\alpha}}{\alpha}\right)},
$$

where $\Gamma(a)$ and $\Gamma(a, b)$ are the gamma and the incomplete gamma functions, respectively.

(iv) Burkert:

$$
\rho_{s}\left(c_{\mathrm{vir}}\right)=\frac{4 \delta_{c} \rho_{\mathrm{crit}}}{3} \frac{c_{\mathrm{vir}}^{3}}{2 \ln \left(1+c_{\mathrm{vir}}\right)+\ln \left(1+c_{\mathrm{vir}}^{2}\right)-2 \tan ^{-1}\left(c_{\mathrm{vir}}\right)} .
$$

\section{References}

[1] Bernabei R, Belli P, Cappella F, Cerulli R, Dai C et al. 2010 Eur.Phys.J. C67 39-49 (Preprint 1002.1028)

[2] Aalseth C E et al. (CoGeNT) 2010 (Preprint 1002.4703)

[3] Fitzpatrick A, Hooper D and Zurek K M 2010 Phys.Rev. D81 115005 (Preprint 1003.0014) 
[4] Chang S, Liu J, Pierce A, Weiner N and Yavin I 2010 JCAP 1008018 (Preprint 1004.0697)

[5] Fitzpatrick A and Zurek K M 2010 Phys.Rev. D82 075004 (Preprint 1007.5325)

[6] Hooper D, Collar J, Hall J and McKinsey D 2010 Phys.Rev. D82 123509 (Preprint 1007.1005)

[7] Schwetz T 2010 (Preprint 1011.5432)

[8] Andreas S, Arina C, Hambye T, Ling F S and Tytgat M H 2010 Phys.Rev. D82 043522 (Preprint 1003.2595)

[9] Barger V, McCaskey M and Shaughnessy G 2010 Phys.Rev. D82 035019 (Preprint 1005.3328)

[10] Fornengo N, Scopel S and Bottino A 2011 Phys.Rev. D83 015001 (Preprint 1011.4743)

[11] Belikov A V, Gunion J F, Hooper D and Tait T M 2010 (Preprint 1009.0549)

[12] Das D and Ellwanger U 2010 JHEP 1009085 (Preprint 1007.1151)

[13] Kappl R, Ratz M and Winkler M W 2011 Phys.Lett. B695 169-173 (Preprint 1010.0553)

[14] Draper P, Liu T, Wagner C E, Wang L T and Zhang H 2011 Phys.Rev.Lett. 106121805 (Preprint 1009.3963)

[15] Kang Z, Li T, Liu T, Tong C and Yang J M 2011 JCAP 1101028 (Preprint 1008.5243)

[16] Mambrini Y 2010 JCAP 1009022 (Preprint 1006.3318)

[17] Akerib D S et al. (CDMS) 2006 Phys. Rev. Lett. 96011302 (Preprint astro-ph/0509259)

[18] Ahmed Z et al. (CDMS-II Collaboration) 2009 (Preprint 0912.3592)

[19] XENON100 Collaboration 2011 (Preprint 1104.2549)

[20] Angle J et al. (XENON) 2008 Phys. Rev. Lett. 100021303 (Preprint arXiv:0706.0039)

[21] EDELWEISS Collaboration 2011 (Preprint 1103.4070)

[22] Angloher G et al. 2008 (Preprint arXiv:0809.1829)

[23] Lebedenko V N et al. 2008 (Preprint arXiv:0812.1150)

[24] Kopp J, Schwetz T and Zupan J 2009 (Preprint 0912.4264)

[25] Savage C, Gelmini G, Gondolo P and Freese K 2011 Phys.Rev. D83 055002 (Preprint 1006.0972)

[26] Trotta R 2008 Contemp.Phys. 49 71-104 (Preprint 0803.4089)

[27] Baltz E A and Gondolo P 2004 JHEP 0410052 (Preprint hep-ph/0407039)

[28] Allanach B and Lester C 2006 Phys.Rev. D73 015013 (Preprint hep-ph/0507283)

[29] Trotta R, Feroz F, Hobson M P, Roszkowski L and Ruiz de Austri R 2008 JHEP 0812024 (Preprint 0809.3792)

[30] Strigari L E and Trotta R 2009 JCAP 0911019 (Preprint 0906.5361)

[31] Bertone G, Cerdeno D G, Fornasa M, de Austri R R and Trotta R 2010 Phys.Rev. D82 055008 (Preprint 1005.4280)

[32] Akrami Y, Savage C, Scott P, Conrad J and Edsjö J 2010 (Preprint 1011.4318)

[33] Vogelsberger M, Helmi A, Springel V, White S D, Wang J et al. 2008 (Preprint 0812.0362)

[34] Ling F S, Nezri E, Athanassoula E and Teyssier R 2010 JCAP 1002012 (Preprint 0909.2028)

[35] Kuhlen M, Weiner N, Diemand J, Madau P, Moore B et al. 2010 JCAP 1002030 (Preprint 0912.2358)

[36] Peter A H 2009 Phys.Rev. D79 103531 (Preprint 0902.1344)

[37] Ullio P and Kamionkowski M 2001 JHEP 0103049 (Preprint hep-ph/0006183)

[38] Belli P, Cerulli R, Fornengo N and Scopel S 2002 Phys.Rev. D66 043503 (Preprint hep-ph/0203242)

[39] Vergados J D, Hansen S H and Host O 2008 Phys. Rev. D77 023509 (Preprint 0711.4895 )

[40] March-Russell J, McCabe C and McCullough M 2008 (Preprint arXiv:0812.1931)

[41] Fairbairn M and Schwetz T 2009 JCAP 0901037 (Preprint arXiv:0808.0704)

[42] Green A M 2010 JCAP 1010034 (Preprint 1009.0916)

[43] Stiff D, Widrow L M and Frieman J 2001 Phys.Rev. D64 083516 (Preprint astro-ph/0106048)

[44] Freese K, Gondolo P, Newberg H J and Lewis M 2004 Phys.Rev.Lett. 92111301 (Preprint astro-ph/0310334)

[45] Savage C, Freese K and Gondolo P 2006 Phys.Rev. D74 043531 (Preprint astro-ph/0607121)

[46] Green A M 2007 JCAP 0708022 (Preprint hep-ph/0703217)

[47] Schneider A, Krauss L and Moore B 2010 Phys.Rev. D82 063525 (Preprint 1004.5432) 
[48] Helm R H 1956 Phys. Rev. 104 1466-1475

[49] Lewin J D and Smith P F 1996 Astropart. Phys. 6 87-112

[50] Binney J and Tremaine S 1987 Galactic Dynamics (Princeton University Press)

[51] Navarro J F, Frenk C S and White S D M 1997 Astrophys. J. 490 493-508 (Preprint astro-ph/9611107)

[52] Ghigna S, Moore B, Governato F, Lake G, Quinn T R et al. 2000 Astrophys.J. 544616 (Preprint astro-ph/9910166)

[53] Einasto J and Haud U 1989 Astronomy and Astrophysics 223 89-106

[54] Graham A W, Merritt D, Moore B, Diemand J and Terzic B 2006 Astron. J. 1322685 (Preprint astro-ph/0509417)

[55] Burkert A 1996 IAU Symp. 171175 (Preprint astro-ph/9504041)

[56] Lisanti M, Strigari L E, Wacker J G and Wechsler R H 2010 (Preprint 1010.4300)

[57] Green A M 2003 Phys. Rev. D68 023004 (Preprint astro-ph/0304446)

[58] Savage C, Gelmini G, Gondolo P and Freese K 2008 (Preprint arXiv:0808.3607)

[59] Loredo T J 1995 81-142 Available from http://bayes.wustl.edu/gregory/articles.pdf

[60] Akerib D S et al. (CDMS) 2004 Phys. Rev. Lett. 93211301 (Preprint astro-ph/0405033)

[61] Ahmed Z et al. (CDMS) 2009 Phys. Rev. Lett. 102011301 (Preprint arXiv:0802.3530)

[62] Ahmed Z et al. (CDMS-II Collaboration) 2011 Phys.Rev.Lett. 106131302 (Preprint 1011.2482)

[63] Akerib D et al. (CDMS Collaboration) 2010 Phys.Rev. D82 122004 (Preprint 1010.4290)

[64] Barbeau P 2009 PhD thesis

[65] Angle J et al. (XENON10) 2009 Phys. Rev. D80 115005 (Preprint 0910.3698)

[66] Aprile E et al. (XENON100 Collaboration) 2011 (Preprint 1103.0303)

[67] Angle J et al. 2011 (Preprint 1104.3088)

[68] Bernabei R et al. (DAMA) 2008 Eur. Phys. J. C56 333-355 (Preprint arXiv:0804.2741)

[69] Bozorgnia N, Gelmini G B and Gondolo P 2010 JCAP 1011019 (Preprint 1006.3110)

[70] Bernabei R et al. 1996 Phys. Lett. B389 757-766

[71] Chagani H, Majewski P, Daw E, Kudryavtsev V and Spooner N 2008 JINST 3 P06003 (Preprint $0806.1916)$

[72] Fushimi K, Ejiri H, Kinoshita H, Kudomi N, Kume K et al. 1993 Phys.Rev. C47 425-428

[73] Smith P, Arnison G, Homer G, Lewin J, Alner G et al. 1996 Phys.Lett. B379 299-308

[74] Navarro J F et al. 2008 (Preprint 0810.1522)

[75] Jimenez R, Verde L and Oh S 2003 Mon.Not.Roy.Astron.Soc. 339243 (Preprint astro-ph/0201352)

[76] Seidel W 2010 talk at IDM, Montpellier, France

[77] CDMS and EDELWEISS Collaborations 2011 (Preprint 1105.3377)

[78] Reid M, Menten K, Zheng X, Brunthaler A, Moscadelli L et al. 2009 Astrophys.J. 700 137-148 (Preprint 0902.3913)

[79] Gillessen S, Eisenhauer F, Trippe S, Alexander T, Genzel R et al. 2009 Astrophys.J. 692 1075-1109 (Preprint 0810.4674)

[80] Smith M C, Ruchti G, Helmi A, Wyse R, Fulbright J et al. 2007 Mon.Not.Roy.Astron.Soc. 379 755-772 (Preprint astro-ph/0611671)

[81] Dehnen W and Binney J 1998 Mon. Not. Roy. Astron. Soc. 298 387-394 (Preprint astro-ph/9710077)

[82] Weber M and de Boer W 2010 Astron.Astrophys. 509 A25 (Preprint 0910.4272)

[83] Salucci P, Nesti F, Gentile G and Martins C 2010 Astron.Astrophys. 523 A83 (Preprint 1003.3101)

[84] Dehnen W, McLaughlin D and Sachania J 2006 Mon.Not.Roy.Astron.Soc. 369 1688-1692 (Preprint astro-ph/0603825)

[85] Sakamoto T, Chiba M and Beers T C 2003 Astron.Astrophys. 397 899-912 (Preprint astro-ph/0210508)

[86] Lewis A and Bridle S 2002 Phys. Rev. D66 103511 (Preprint astro-ph/0205436)

[87] Lewis A and Bridle S CosmoMC notes http://cosmologist.info/notes/CosmoMC.pdf 
[88] Metropolis N, Rosenbluth A W, Rosenbluth M N, Teller A H and Teller E 1953 Journal of Chemical Physics 21 1087-1092

[89] Hastings W 1970 Biometrika 97-109

[90] de Austri R R, Trotta R and Feroz F SuperBayeS package http://www.superbayes.org/

[91] Hamann J, Hannestad S, Raffelt G and Wong Y Y 2007 JCAP 0708021 (Preprint 0705.0440)

[92] Helene O 1983 Nucl.Instrum.Meth. 212319

[93] Zech G 1989 Nucl.Instrum.Meth. A277 608

[94] Cousins R D and Highland V L 1992 Nucl.Instrum.Meth. A320 331-335 revised version

[95] Frandsen M T, Kahlhoefer F, March-Russell J, McCabe C, McCullough M et al. 2011 (Preprint 1105.3734)

[96] Farina M, Kadastik M, Pappadopulo D, Pata J, Raidal M et al. 2011 (Preprint 1104.3572)

[97] Collar J 2011 STSI, 2011 May symposium, Baltimore, Mariland

[98] Graham P W, Harnik R, Rajendran S and Saraswat P 2010 Phys.Rev. D82 063512 (Preprint 1004.0937) 